\title{
Confronting the trans-Planckian question \\ OF INFLATIONARY COSMOLOGY \\ WITH DISSIPATIVE EFFECTS
}

\author{
Renaud Parentani \\ Laboratoire de Physique Théorique, UMR 8627, \\ Bât. 210, Université Paris-Sud 11, \\ 91405 Orsay Cedex, France
}

\begin{abstract}
We provide a class of QFTs which exhibit dissipation above a threshold energy, thereby breaking Lorentz invariance. Unitarity is preserved by coupling the fields to additional degrees of freedom (heavy fields) which introduce the rest frame. Using the Equivalence Principle, we define these theories in arbitrary curved spacetime. We then confront the trans-Planckian question of inflationary cosmology. When dissipation increases with the energy, the quantum field describing adiabatic perturbations is completely damped at the onset of inflation. However it still exists as a composite operator made with the additional fields. And when these are in their ground state, the standard power spectrum obtains if the threshold energy is much larger that the Hubble parameter. In fact, as the energy redshifts below the threshold, the composite operator behaves as if it were a free field endowed with standard vacuum fluctuations. The relationship between our models and the Brane World scenarios studied by Libanov and Rubakov displaying similar effects is discussed. The signatures of dissipation will be studied in a forthcoming paper.
\end{abstract}




\section{Introduction and presentation of the settings}

Even though relativistic QFT provides an excellent description of particle physics, being noncompact, Lorentz symmetry can only be tested up to a certain energy scale. Thus one cannot exclude that some unknown high energy processes break the invariance under boosts, thereby introducing a threshold energy $\Lambda_{L V}$, and a preferred frame. It is therefore of interest to determine what would be the signatures when this possibility is realized [1].

This question is particularly relevant for inflationary cosmology because primordial density fluctuations arise from vacuum fluctuations which had very short wave lengths (very large proper frequencies) at the onset of inflation[2]. Indeed, the initial frequencies of the modes we observe today in the CMB anisotropies were all larger than

$$
\Omega_{\text {in }}=H e^{N_{\text {extra }}},
$$

where $H$ is the value of the Hubble parameter during inflation, and where $N_{\text {extra }}$ is the number of e-folds from the onset of inflation till $t_{0}$, the moment when the (comoving) scale of our visible universe exited the Hubble radius. (The total number of e-folds is thus $N_{0}+N_{\text {extra }}$ where $N_{0} \sim 60$ is the number of e-folds from $t_{0}$ till the end of inflation.) Irrespectively of the inflationary scenario, $\Omega_{i n}$ is thus larger than the Planck mass $M_{P l}$ when $N_{\text {extra }}>\ln M_{P l} / H$. Since $H$ should be of the order of $10^{-5} M_{P l}$, the initial frequencies of observable modes were all trans-Planckian when $N_{\text {extra }}>5 \ln 10 \sim 12$. Moreover, since in most scenarios $N_{\text {extra }} \gg 12$, $\Omega_{\text {in }}$ was generically much larger than the Planck mass.

In the absence of Quantum Gravity, there is no understanding of the nature of the degrees of freedom at these scales: we neither know what is their Hilbert space, nor how they propagate, and this, because the notions of differential geometry used when describing (quantum) fields in expanding universes might not apply at very short distances. In particular eq. (1) which is based on a relativistic mode equation in an accelerating universe might loose its validity above a certain Ultra-Violet scale $\Lambda_{L V}$. Since this scale might significantly differ from the Planck mass, we shall keep it distinct from the Planck mass. We shall also use the abbreviations $L I$ and $L V$ for Lorentz Invariance and Lorentz Violation respectively.

In this context, it is instructive to phenomenologically parameterize the deviations from $L I$ and determine their signatures on inflationary spectra. This line of thought has been proposed in the context of Hawking radiation from black holes, wherein the asymptotic quanta also arise from vacuum fluctuations with exponentially growing frequencies [3, 4]. The deviations from $L I$ have been characterized by non-linear dispersion relations imposed in a particular rest frame,

$$
\Omega^{2}=F_{n}\left(p^{2}\right)=p^{2}\left(1 \pm p^{2 n} / \Lambda_{L V}^{2 n}+O\left(p^{2 n+2}\right)\right),
$$

where $\Omega$ and $p$ are respectively the frequency and the norm of the spatial momentum measured in that frame. The first deviation is characterized by a power of $p / \Lambda_{L V}$ and the sign defining super-luminous $(+)$ and sub-luminous (-) cases. When $\Lambda_{L V}$ is much larger than Hawking temperature, and when the frame is freely falling, it was shown that the asymptotic properties of Hawking radiation are unmodified, even though the near horizon propagation is radically modified when $\Omega$ is larger than $\Lambda_{L V}$. The robustness of asymptotic properties relies on the 
adiabatic character of the evolution of the vacuum (ground) state [5]. The same program was then applied to the inflationary spectra [6, 7], and similar results were obtained because, in this case as well, the ground state adiabatically evolves when $\sigma=H / \Lambda_{L V} \ll 1$ [8]. However, so far, only dispersive models have been systematically studied.

The aim of the present work is to extend this analysis to dissipative models. To this end, we shall first construct QFT displaying dissipative effects in the UV. Indeed, unlike dispersion, dissipation requires enlarged dynamical settings because if one tries to introduce dissipation from the outset in eq. (2), one looses both unitarity and predictability. To preserve them, we shall work with Hamiltonian theories in which dissipative effects are caused by interactions with additional fields. Doing so, we shall establish that dissipative effects are generic. That is, when starting with a Lagrangian in which $L I$ is broken in the UV, the effective theory unavoidably develops dissipation above a certain energy scale, simply because nothing can prevent this. (With relativistic QFT instead, LI did prevent it).

Since we want to construct generalized QFT, we should decide what to keep. First, we want to preserve the unitarity of the description because the calculation of the power spectrum of (adiabatic) density fluctuations [2] rests on the identification of a scalar field, hereafter called $\phi$, which obeys Equal Time Commutators (ETC). This identification is necessary to fix the rms amplitude of density fluctuations in the vacuum. Explicitely the power spectrum is given

$$
P_{p}(t) \equiv 4 \pi p^{3} \int\left(\frac{d x}{2 \pi}\right)^{3} e^{i \mathbf{p x}} G_{a}(t, \mathbf{x} ; t, \mathbf{0}),
$$

where $G_{a}$ is the anti-commutator of $\phi$ evaluated in the asymptotic (Bunch-Davies) vacuum much after horizon exit. In the absence of dissipation, $P_{p}$ can be deduced from the norm of $\phi_{p}^{i n}(t)$, the Fourier mode of $\phi$ with asymptotic positive frequency. However in the presence of dissipation, this notion of (free) mode disappears. Hence the knowledge of $G_{a}$ becomes necessary since eq. (3) gives the only way to obtain the power spectrum.

To get dissipation we shall thus introduce additional degrees of freedom, hereafter called $\Psi$. Then the whole system $(\phi+\Psi)$ will evolve unitarily, by construction. This guarantees that the ETC of $\phi$ is exactly preserved (in a non trivial way since $\phi$ undergoes dissipation). Moreover, the (dressed) 2pt function of $\phi$ will be given by the usual QM trace

$$
G_{W}(x, y)=\operatorname{AR}\left[\hat{\rho}_{T} \hat{\phi}(x) \hat{\phi}(y)\right]
$$

where $\hat{\rho}_{T}$ is the initial matrix density of the total system, where $\hat{\phi}(x)$ is the Heisenberg field operator evolved with the time ordered exponential of the total Hamiltonian, and where the trace is taken over both $\Psi$ and $\phi$. The anti-commutator $G_{a}$ determining the power spectrum in eq. (3) is simply the symmetric part of $G_{W}$.

Our second requirement concerns the properties of dissipative effects. When considering the theory in vacuum and in Minkowski space-time, we impose that these effects preserve the stationary, the homogeneity, and isotropy of flat space-time. These requirements define a preferred frame which is inertial and globally defined. Then irrespectively of the choice of $\Psi$ and $\Psi-\phi$ interactions, the Fourier transform of the retarded Green function, $G_{r}(x, y)=$ 
$\theta\left(t_{x}-t_{y}\right) 2 \operatorname{Im} G_{W}(x, y)$, is of the form

$$
G_{r}(\omega, \vec{p})=\frac{1}{\left(-\omega^{2}+p^{2}+\Sigma_{r}(\omega, p)\right)} .
$$

In the true vacuum, at the level of the 2 pt functions, the dissipative (dispersive) effects are thus completely characterized by the imaginary and odd part in $\omega$ (real and even part) of the self energy $\Sigma_{r}(\omega, p)$.

In these expressions, the energy $\omega$ and the momentum square $p^{2}$ are defined in the preferred frame. The novelty is that $\Sigma_{r}$ is a function of $\omega$ and $p$ separately, and not only a function of the relativistic invariant $\omega^{2}-p^{2}$ as it is the case in $L I$ QFT. Therefore dissipation can become significant above a critical energy on the mass shell, i.e. along the minima of the denominator of eq. (5); a possibility forbidden in $L I$ theories. For instance, one verifies that the following self-energies induce significant dissipation only above $\Lambda_{L V}$

$$
\operatorname{Im} \Sigma_{r}^{(n)}(\omega, p)=-\frac{\omega}{\Lambda_{L V}} p^{2}\left(\frac{p^{2}}{\Lambda_{L V}^{2}}\right)^{n} .
$$

With this equation we have identified the relevant (lowest order) quantity governing dissipation, the equivalent of the first order deviation in eq. (2). In the body of the paper, we shall provide Lagrangians of $\Psi$ and $\phi$ which give this class of self-energies labeled by $n$. Rather than focusing on a particular case, we shall describe the whole class of dissipative behaviors (at the level of 2-point functions). We shall thus follow a phenomenological approach based on minimal assumptions (i.e. unitarity), rather than some "inspired" approach (e.g. by string theory, or branes scenarios [9, 10]) which would lead to a particular sub-class of models.

Our third requirement concerns the extension of our models from Minkowski space to curved space-times. To define our QFT in curved space, we simply implement the Equivalence Principle (EP). It fixes the action density our models to be the covariantized version of that we had in Minkowski space (up to some non-minimal coupling). To perform the covariantization, it is useful to characterize the preferred frame in a coordinate invariant way by $l^{\mu}$, a unit time-like vector field [11]. In terms of this vector, $\omega$ and $p^{2}$ are given by

$$
\omega \equiv l^{\mu} p_{\mu}, \quad p^{2} \equiv \perp^{\mu \nu} p_{\mu} p_{\nu}
$$

where $\perp^{\mu \nu} \equiv g^{\mu \nu}+l^{\mu} l^{\nu}$ is the (positive definite) metric in the spatial sections orthogonal to $l^{\mu}$. The covariant action will be a sum of scalar functions of the four local fields $\phi, \Psi, g_{\mu \nu}$ and $l^{\nu}$ which reduces to the Minkowski model in the zero curvature limit. Very importantly for us, we shall see that the EP guarantees the adiabaticity of the evolution of the (interacting) ground state as long the gradients of the metric are much smaller than the UV scale $\Lambda_{L V}$.

For the interested reader, we mention that additional comments which place the present work in broader contexts can be found in the introduction of [12]. These comments concern Quantum Gravity and the description of "mode creation" in expanding universes. 


\section{Dissipation in Minkowski space from $L V$ effects}

In this Section, we provide a class of models defined in Minkowski spacetime which exhibit dissipative effects above a certain energy scale $\Lambda_{L V}$. Stationarity, homogeneity and isotropy will be exactly preserved. Therefore, the only invariance of relativist QFT which is broken is that under boosts. These theories define a preferred rest frame which is globally defined, as it is the case in FLRW space-times. For the simplicity of the presentation, we shall first work in the preferred frame. At the end of this Section we shall covariantize our action and generalize it to curved spacetime.

\subsection{Free field settings}

We start with a brief presentation of the free field quantization to introduce notations and to point out what are the properties which will be lost in the presence of dissipation.

The action of our free massless field $\phi$ is the usual one:

$$
S_{\phi}=\frac{1}{2} \int d t d^{3} x\left(\partial_{t} \phi^{2}-\partial_{\mathbf{x}} \phi \cdot \partial_{\mathbf{x}} \phi\right)
$$

where $t$ and $\mathbf{x}$ are Cartesian coordinates. Due to the homogeneity of space, the equation of motion can be analyzed mode by mode:

$$
\phi(t, \mathbf{x})=\int \frac{d^{3} p}{(2 \pi)^{3 / 2}} e^{i \mathbf{p} \cdot \mathbf{x}} \phi_{\mathbf{p}}(t)
$$

The Fourier mode $\phi_{\mathbf{p}}(t)$ obeys

$$
\left(\partial_{t}^{2}+\omega_{p}^{2}\right) \phi_{\mathbf{p}}=0
$$

where $\omega_{p}^{2}=p^{2}=\mathbf{p} \cdot \mathbf{p}$ is the standard relativistic dispersion relation. Notice that eq. (10) is second order, homogeneous (no source term), and time reversible (no odd power of $\partial_{t}$ ), three properties we shall loose when introducing interactions breaking $L I$.

In homogeneous space-times, the Equal Time Commutator between the Heisenberg field operator and its momentum implies that the mode operator (q-number) $\phi_{\mathbf{p}}(t)$ obeys

$$
\left[\phi_{\mathbf{p}}(t), \partial_{t} \phi_{\mathbf{p}^{\prime}}^{\dagger}(t)\right]=i \delta^{3}\left(\mathbf{p}-\mathbf{p}^{\prime}\right)
$$

When decomposing this operator as

$$
\phi_{\mathbf{p}}(t)=a_{\mathbf{p}} \phi_{p}(t)+a_{-\mathbf{p}}^{\dagger} \phi_{p}^{*}(t)
$$

where the destruction and creation operators satisfy the usual commutators

$$
\left[a_{\mathbf{p}}, a_{\mathbf{p}^{\prime}}^{\dagger}\right]=\delta^{3}\left(\mathbf{p}-\mathbf{p}^{\prime}\right), \quad\left[a_{\mathbf{p}}, a_{\mathbf{p}^{\prime}}\right]=0,
$$

eq. (11) is verified because the Wronskian of the positive frequency (c-number) mode

$$
\phi_{p}(t)=e^{-i \omega_{p} t} /\left(2 \omega_{p}\right)^{1 / 2},
$$


is constant (and conventionally taken to be unity).

Had an odd term like $\gamma \partial_{t}$ be present in eq. (10) the constancy of the Wronskian would have been lost. Hence the possibility of realizing the ETC (11) with the help of eq. (13) would have been lost as well. This already indicates that, unlike dispersive (real) effects, dissipative effects require more general settings than the above.

\subsection{Interacting models breaking $L I$, general properties}

We now introduce additional degrees of freedom, here after collectively named $\boldsymbol{\Psi}$, which induce dissipation above the energy $\Lambda_{L V}$. We shall work with a particular class of models in order to get an exact (non-perturbative) expression for the two-point function of eq. (4). Before introducing these models, we derive general results valid for all unitary QFT's possessing dissipative effects above $\Lambda_{L V}$ in the ground state (the interacting vacuum).

We assume that the total action decomposes as

$$
S_{T}=S_{\phi}+S_{\Psi}+S_{\phi, \Psi}
$$

where the first action is that of eq. (8), the second one governs the evolution of the $\boldsymbol{\Psi}$ fields, and the last one the coupling between $\phi$ and these new fields. We also impose that the last two actions preserve the homogeneity and isotropy of Minkowski space but break the invariance under boosts. From now on, the coordinates $t, \mathbf{x}$ are at rest with respect to the preferred frame defined by $S_{\Psi}+S_{\phi, \Psi}$, i.e., $\partial_{t} \equiv l^{\mu} \partial_{\mu}$ in the covariant notation of eq. (17).

When the state of such system is homogeneous, the Fourier transform of the 2pt function of eq. (4) is of the form

$$
\begin{aligned}
G_{\mathbf{p}, \mathbf{p}^{\prime}}\left(t, t^{\prime}\right) & =\operatorname{Tr}\left[\hat{\rho}_{T} \hat{\phi}_{\mathbf{p}}(t) \hat{\phi}_{\mathbf{p}^{\prime}}^{\dagger}\left(t^{\prime}\right)\right] \\
& =G_{W}\left(t, t^{\prime} ; p\right) \delta^{3}\left(\mathbf{p}-\mathbf{p}^{\prime}\right)
\end{aligned}
$$

At this point, an important remark should be made. In the presence of interactions, the notion (and the usefulness) of the time-dependent modes of eq. (14) has disappeared whereas the function $G_{W}\left(t, t^{\prime} ; p\right)$ of eq. (16) is always well-defined, for all choices of $\boldsymbol{\Psi}$ and $S_{\boldsymbol{\Psi}}+S_{\phi, \boldsymbol{\Psi}}$.

When the situation is stationary, $G_{W}\left(p ; t, t^{\prime}\right)$ further simplifies in the frequency representation:

$$
G_{W}\left(t, t^{\prime} ; p\right)=\int \frac{d \omega}{2 \pi} e^{-i \omega\left(t-t^{\prime}\right)} G_{W}(\omega, p) .
$$

When working in the ground state, one finds the simplest case, because whatever the $\boldsymbol{\Psi}$ fields may be, the Fourier transform of the time ordered (Feynman) propagator,

$$
2 G_{F}=\operatorname{Re} G_{W}+i \operatorname{Im} G_{W} \operatorname{sign}\left(t-t^{\prime}\right)
$$

is always of the same form as $G_{r}$ in eq. (5) , and therefore characterized by a single (complex) function $\Sigma_{F}(\omega, p)$. When restricting attention to Gaussian models, $\Sigma_{F}(\omega, p)$ is given by a 1-loop calculation, whereas in general, it contains a series of 1PI graphs. 
In non-vacuum states and in non-stationary situations, the $2 \mathrm{pt}$ functions and self-energies have a more complicated structure [20]. To understand this structure it is useful to study separately the commutator $G_{c}=i \operatorname{Im} G_{W}$ and the anti-commutator $G_{a}=\operatorname{Re} G_{W}$.

Let us conclude with two remarks. First, the effective dispersion relation of $\phi$ is a posteriori defined by the poles of eq. (5) which are governed by $\Sigma(\omega, p)$ [13, 14]. In this way, nontrivial dispersion relations arise from dynamical processes rather than from being introduced from the outset. The present work therefore provides physical foundations (and restrictions, as later discussed) to the kinematical approach which is usually adopted [1]. Second, from analyzing dynamical models, we shall see that, even in the vacuum, on-shell dissipative effects (i.e. dissipation arising along the minima of the denominator of eq. (5)) are unavoidable when $L I$ is broken in the UV by the action $S_{\Psi}+S_{\phi, \Psi}$, in complete opposition with the fact that on-shell dissipation is forbidden when working (in the vacuum) with $L I$ actions.

\subsection{Gaussian models}

To simplify the calculation of $\Sigma(\omega, p)$ and to get non perturbative expressions, we assume that the action $S_{T}$ is quadratic in all field variables. At first sight, this could be considered as an artificial hypothesis. However, it should be recalled that we are not after computing $\Sigma$ from first principles. Rather we aim to compute the signatures of the power spectrum (3) given the properties of $\Sigma$, following the approach adopted in [3, 5, 6, 7].

Given that we are preserving the homogeneity of Minkowski space, the Gaussian assumption implies that the total action splits as

$$
S_{T}=\int d^{3} p S_{T}(\mathbf{p})
$$

where each action $S_{T}(\mathbf{p})$ depends only on $\phi_{\mathbf{p}}$ and the $\mathbf{p}$-th Fourier component of $\boldsymbol{\Psi}$. The structure of these actions is

$$
\begin{aligned}
S_{T}(\mathbf{p})= & \frac{1}{2} \int d t \phi_{\mathbf{p}}^{*}\left(-\partial_{t}^{2}-\omega_{p}^{2}\right) \phi_{\mathbf{p}}+\frac{1}{2} \Sigma_{i} \int d t \Psi_{i}^{*}(\mathbf{p})\left(-\partial_{t}^{2}-\Omega_{i}^{2}(p)\right) \Psi_{i}(\mathbf{p}) \\
& +\Sigma_{i} \int d t g_{i}(p) \phi_{\mathbf{p}} \Psi_{i}^{*}(\mathbf{p})
\end{aligned}
$$

where $i$ is a discrete (or continuous) index, where $\Omega_{i}(p)$ is the energy of the quanta of the oscillators $\Psi_{i}(\mathbf{p})$, and where $g_{i}(p)$ is the coupling constant at fixed $p, i$. Since $\phi_{\mathbf{p}}^{\dagger}=\phi_{-\mathbf{p}}$, $\Psi_{i}^{\dagger}(\mathbf{p})=\Psi_{i}(-\mathbf{p}), S_{T}(\mathbf{p})+S_{T}(-\mathbf{p})$ is real. Instead of choosing a priori one model, it is more instructive to solve the equations of motion without specifying the set of $\Psi_{i}(\mathbf{p})$, their energy $\Omega_{i}(p)$ and their coupling $g_{i}(p)$. We shall choose them in due course. To preserve stationarity in Minkowski space, the $\Omega$ 's and the $g$ 's must be time independent. When $\Omega_{i}^{2}(p) \neq M_{i}^{2}+p^{2}$, the kinetic action of $\Psi_{i}(\mathbf{p})$ breaks $L I$ and defines the preferred frame. On the contrary when $\Omega_{i}^{2}(p)=M_{i}^{2}+p^{2}$ the preferred frame is only defined by $S_{\phi \Psi}$ through the $p$-dependence of the coupling functions $g_{i}(p)$.

Models of this type have been used for different purposes. They have been introduced (in their continuous version) to study non-pertubatively atomic transitions see [15] and refs. 
therein, see also [16, 17] for an application to the Unruh effect. They have been used in Quantum Optics [18], to model quantum Brownian motion, and to study decoherence effects [19]. Depending on the aims, they can be solved and analyzed by means of different methods. In what follows we shall use the simplest approach based on Heisenberg picture 1 Since we shall work at zero temperature, this approach offers a simple characterization of the state of the system in terms of the ground states of the free modes before $\phi-\Psi$ interactions are turned on.

To prepare the application to inflation, we shall treat $\omega_{p}^{2}, \Omega_{i}^{2}$ and $g_{i}$ as arbitrary functions of time (in cosmology these quantities become time dependent through their dependence in the scale factor $a(t))$. The equations of motion are

$$
\begin{aligned}
& \left(\partial_{t}^{2}+\omega_{p}^{2}\right) \phi_{\mathbf{p}}=\Sigma_{i} g_{i}(p) \Psi_{i}(\mathbf{p}) \\
& \left(\partial_{t}^{2}+\Omega_{i}^{2}\right) \Psi_{i}(\mathbf{p})=g_{i}(p) \phi_{\mathbf{p}}
\end{aligned}
$$

The general solution of the second equation reads

$$
\Psi_{i}(\mathbf{p}, t)=\Psi_{i}^{o}(\mathbf{p}, t)+\int d t^{\prime} R_{i}^{o}\left(t, t^{\prime} ; p\right) g_{i}\left(t^{\prime} ; p\right) \phi_{\mathbf{p}}\left(t^{\prime}\right)
$$

where $\Psi_{i}^{o}(\mathbf{p}, t)$ is a free solution which depends on initial conditions imposed on $\Psi_{i}(\mathbf{p})$. The second term contains $R_{i}^{o}\left(t, t^{\prime} ; p\right)$, the (free) retarded Green function of $\Psi_{i}(\mathbf{p})$. It obeys

$$
\left(\partial_{t}^{2}+\Omega_{i}^{2}(p)\right) R_{i}^{o}\left(t, t^{\prime} ; p\right)=\delta\left(t-t^{\prime}\right)
$$

and vanishes for $t<t^{\prime}$. Injecting eq. (23) in eq. (21) one gets

$$
\left(\partial_{t}^{2}+\omega_{p}^{2}\right) \phi_{\mathbf{p}}=\Sigma_{i} g_{i}(t ; p) \Psi_{i}^{o}(\mathbf{p}, t)+\Sigma_{i} g_{i}(t ; p) \int d t^{\prime} R_{i}^{o}\left(t, t^{\prime} ; p\right) g_{i}\left(t^{\prime} ; p\right) \phi_{\mathbf{p}}\left(t^{\prime}\right) .
$$

The solution of this equation has always the following structure

$$
\phi_{\mathbf{p}}(t)=\phi_{\mathbf{p}}^{d}(t)+\int d t^{\prime} G_{r}\left(t, t^{\prime} ; p\right)\left[\Sigma_{i} g_{i}\left(t^{\prime} ; p\right) \Psi_{i}^{o}\left(\mathbf{p}, t^{\prime}\right)\right]
$$

The first term is the "decaying" solution. It contains all the information about the initial condition of $\phi_{\mathbf{p}}$. The second term is the "driven" solution. It is governed by the initial conditions of $\Psi_{i}(\mathbf{p})$ and by the (dressed) retarded Green function, the solution of

$$
\int d t^{\prime}\left[\delta\left(t-t^{\prime}\right)\left(\partial_{t^{\prime}}^{2}+\omega_{p}^{2}\right)-\Sigma_{i} g_{i}(t ; p) R_{i}^{o}\left(t, t^{\prime} ; p\right) g_{i}\left(t^{\prime} ; p\right)\right] G_{r}\left(t^{\prime}, t_{1} ; p\right)=\delta\left(t-t_{1}\right)
$$

Notice that $\phi_{\mathbf{p}}^{d}\left(t^{\prime}\right)$ is an homogeneous solution of this equation. Therefore the evolution of both $\phi^{d}$ and $G_{r}$ fully takes into account, through the non-local term in the above bracket, the

\footnotetext{
${ }^{1}$ Even though legitimate, we shall not use the general methods (Influence Functional, Master Equation, Closed Time Path Integral) [18] which have been developed to study "open quantum systems" because they somehow hide the simplicity of the present models. Moreover we are planning (in a subsequent work) to study the correlations between $\phi$ and $\Psi_{i}$. Therefore we shall treat $\phi$ and $\Psi_{i}$ on equal footing as in [15, 16, 17].
} 
back-reaction due to the coupling to the $\Psi_{i}$. In Gaussian models, it is quadratic in $g_{i}$. Hence $\phi^{d}$ and $G_{r}$ are series containing all powers of $g_{i}$. Moreover since $g_{i}(t ; p)$ are arbitrary functions of $p$ and $t$, at this point, there is no reason to consider non-Gaussian models.

To conclude this subsection, we notice that eq. (26) also furnishes the exact solution for the (Heisenberg) mode operator $\phi_{\mathbf{p}}(t)$ because the equations we solved were all linear. Since the power spectrum in inflation is obtained from vacuum fluctuations, instead of further analyzing the time dependence of the mode (as one would do in classical terms), it is more relevant to study the correlation functions of $\phi_{\mathbf{p}}(t)$.

\subsection{Structure of two-point correlation functions}

This sub-section mainly contains well-known results which follow from the linearity of eq. (26). The key result we shall later use is given in eq. (31).

Since our models are Gaussian, the 2 pt function of eq. (16) governs all observables built with the Heisenberg field operator $\phi$. To analyse it, as already mentioned, it is appropriate to study separately the commutator and the anti-commutator.

We start with the simple part, the commutator

$$
G_{c}\left(t, t^{\prime} ; p\right) \delta^{3}\left(\mathbf{p}-\mathbf{p}^{\prime}\right) \equiv \operatorname{Tr}\left[\rho_{T}\left[\phi_{\mathbf{p}}(t), \phi_{\mathbf{p}^{\prime}}^{\dagger}\left(t^{\prime}\right)\right]_{-}\right] .
$$

From eq. (26) one sees that it decomposes into two terms, one due to the non-commuting character of $\phi^{d}$, the other due to that of $\Psi_{i}^{0}$. In addition, since both commutators are cnumbers, it is independent of $\rho_{T}$, the state of the system. Hence, for all Gaussian models, one has

$$
G_{c}\left(t, t^{\prime} ; p\right)=\left[\phi^{d}(t), \phi^{d}\left(t^{\prime}\right)\right]_{-}+\iint d t_{1} d t_{2} G_{r}\left(t, t_{1}\right) G_{r}\left(t^{\prime}, t_{2}\right) D\left(t_{1}, t_{2}\right)
$$

where the "dissipative" kernel $D\left(t_{1}, t_{2}\right)$ is given by

$$
D\left(t_{1}, t_{2}\right)=\Sigma_{i} \Sigma_{j} g_{i}\left(t_{1}\right) g_{j}\left(t_{2}\right)\left[\Psi_{i}^{o}\left(t_{1}\right), \Psi_{j}^{o}\left(t_{2}\right)\right]_{-}=\Sigma_{i} g_{i}\left(t_{1}\right) G_{c, i}^{o}\left(t_{1}, t_{2}\right) g_{i}\left(t_{2}\right) .
$$

Notice how this kernel combines the coupling $g_{i}$ and the non-commuting properties of $\Psi_{i}$.

The next property of $G_{c}$ is more relevant. To all orders in $g_{i}$ and for all sets of $g_{i}, \Omega_{i}$ (even with arbitrary time dependence), one obtains

$$
\left.i \partial_{t} G_{c}\left(t, t^{\prime} ; p\right)\right|_{t=t^{\prime}}=1 .
$$

This identity corresponds to the ETC of eq. (11). The 1 on the $r h s$ is guaranteed by the Hamiltonian character of the evolution of the entire system $\phi+\Psi$. It is therefore this equation which replaces the constancy of the Wronskian that was relevant in the case of free evolution.

Eq. (31) is crucial for us for two reasons. First, since the operator $\phi^{d}(t)$ in eq. (26) decays in $t-t_{\text {in }}$ where $t_{\text {in }}$ is the moment when the interactions are turn on -because it is an homogeneous solution of eq. (27) - the first term in eq. (29) decays as exp $-\gamma\left(t+t^{\prime}-2 t_{i n}\right)$. Therefore when $\gamma\left(t+t^{\prime}-2 t_{i n}\right) \gg 1$, the non-commuting properties $\phi_{\mathbf{p}}$ are entirely due to those of the environment degrees of freedom, $\Psi_{i}^{o}$. Secondly, these sum up exactly to 1 , as if the driven term of eq. (26) were a canonical degree of freedom. 
We now analyze the anti-commutator,

$$
G_{a}\left(t, t^{\prime} ; p\right) \delta^{3}\left(\mathbf{p}-\mathbf{p}^{\prime}\right) \equiv \operatorname{Tr}\left[\rho_{T}\left\{\phi_{\mathbf{p}}(t), \phi_{\mathbf{p}^{\prime}}^{\dagger}\left(t^{\prime}\right)\right\}_{+}\right] .
$$

When the (initial) density matrix factorizes, $\rho_{T}=\rho_{\phi} \rho_{\Psi}$, as it is the case in the "free" vacuum before the interactions are turned on, $G_{a}$ also splits into two terms,

$$
G_{a}\left(t, t^{\prime} ; p\right)=\operatorname{Tr}\left[\rho_{\phi}\left\{\phi^{d}(t), \phi^{d}\left(t^{\prime}\right)\right\}_{+}\right]+\iint d t_{1} d t_{2} G_{r}\left(t, t_{1}\right) G_{r}\left(t^{\prime}, t_{2}\right) N\left(t_{1}, t_{2}\right)
$$

The first term only depends on the initial state of $\phi$. Similarly, the driven term only depends on the state of the environment through the "noise" kernel

$$
N\left(t_{1}, t_{2}\right)=\Sigma_{i} \Sigma_{j} \operatorname{Tr}\left[\rho_{\Psi}\left\{g_{i}\left(t_{1}\right) \Psi_{i}^{o}\left(t_{1}\right), g_{j}\left(t_{2}\right) \Psi_{j}^{o}\left(t_{2}\right)\right\}_{+}\right] .
$$

As for the commutator, in the presence of dissipation, the first term exponentially decays, expressing the progressive erasing on the information contained in the initial state of $\phi$. At late times therefore it is the state of $\Psi$ which fixes the anti-commutator of $\phi$. This allows to remove the restriction that initially the density matrices factorizes. If one is interested by the late time behavior, only $N$ matters.

In brief, we have recalled two important results. First, at late time, the Heisenberg field $\phi$ reduces to its driven term, the second term of eq. (26), since both its commutator and anti-commutator are determined by those of $\Psi_{i}^{o}$. Second, only two (real) kernels determined by the environment govern the two-point functions of $\phi$, namely $D$ and $N$ of eqs. (30, 34). Therefore the set of environments (Gaussian or not Gaussian) possessing the same kernels will give rise to the same $2 \mathrm{pt}$ functions. Hence they should be viewed as forming an equivalent class. The degeneracy can be lifted by considering correlations with observables containing the operators $\Psi_{i}$, or higher order correlations functions of $\phi$ (for non-Gaussian environments), two possibilities we shall not discuss in this paper.

To compute $G_{c}$ and $G_{a}$, two different routes can be adopted. When $g_{i}(p)$ and $\Omega(p)$ are constant, one should work in Fourier transform because the equations can be algebraically solved, in full generality. Instead when $g_{i}(p)$ and/or $\Omega(p)$ are time-dependent, as it will be the case in expanding universes and in curved space-times, it is appropriate (but not necessary) to exploit the above mentioned degeneracy by choosing the $\Psi_{i}$ and their frequencies $\Omega_{i}^{2}(p)$ so as to simplify the time dependence of the equations. In the text, we proceed with time dependent approach. In Appendix A, we present the Fourier analysis which is straightforward. We invite the reader unfamiliar with the Quantum Mechanical treatment of dissipation to read it.

\subsection{Time dependent settings}

In Appendix B, we provided a class of models characterized by the power of $p / \Lambda_{L V}$ which specifies how dissipative effects grow, see eq. (85). This class covers the general case and can be used as a template to study the consequences of dissipative effects. In addition, as noticed after eq. (76), the dissipative effects are governed by $D=\Sigma_{i} g_{i}^{2} R_{i}^{o}$. Therefore all environments delivering the same kernel give rise to the same (stationary) phenomenology. 
In this Section we exploit this freedom having in mind the transposition of our model from Minkowski space to cosmological, and thus time-dependent, metrics. Therefore the selected models should possess two-point functions with simple properties when expressed in the terms of time (as opposed to Fourier components). The core of the problem is that $G_{a}$ depends, see eq. (33), on the retarded Green function of $\phi$ which is not known. Indeed $G_{r}$ is only implicitly defined as a solution of eq. (27) which is, in general, a non-local differential equation. We are thus led to choose $\Psi$ in order for this equation to be local. This implies that the retarded Green function of $\Psi$ appearing in eq. (27) be proportional to $\delta\left(t-t^{\prime}\right)$. (Since this requirement concerns the Green function of the environment it does not restrict the phenomenology of $\phi$.)

Given this aim, we select the models defined by the action

$$
\begin{aligned}
S_{T}^{(n)}(\mathbf{p})= & \frac{1}{2} \int d t \phi_{\mathbf{p}}^{*}\left(-\partial_{t}^{2}-\omega_{p}^{2}\right) \phi_{\mathbf{p}}+\frac{1}{2} \int d t \int_{-\infty}^{\infty} d k \Psi^{*}(\mathbf{p}, k)\left(-\partial_{t}^{2}-\left(\pi \Lambda_{L V} k\right)^{2}\right) \Psi(\mathbf{p}, k) \\
& +g \Lambda_{L V} \int d t \int_{-\infty}^{\infty} d k\left(\frac{p}{\Lambda_{L V}}\right)^{n+1} \phi_{\mathbf{p}} \partial_{t} \Psi^{*}(\mathbf{p}, k)
\end{aligned}
$$

In $S_{\phi \Psi}$ have factorized out a factor of $\Lambda_{L V}$ so that the coupling constant $g$ is dimensionless.

When compared with the action of eq. (20), we have replaced the discrete index $i$ by the integral over the dimensionless variable $k$. As recalled in Appendix A, the spectrum of the environment must be continuous to have proper dissipation, see discussion after eq. (76). The variable $k$ can be viewed as a momentum, in the units of $\Lambda_{L V}$, in a flat extra spatial dimension. The relationship with some Brane World Scenarios in then clear [9, 10]. In the 'atomic' version of this model [16, 17] which has inspired us, the radiation field $\Psi$ is a massless 2 dimensional field propagating in the dimension associated with $k$.

We have also introduced an additional time derivative acting $\Psi$ in $S_{\phi, \Psi}$. This choice leads to the above mentioned $\delta\left(t-t^{\prime}\right)$. Indeed, on the one hand, taking account this extra derivative, the continuous character of $k$, and the fact that $g$ is independent of $k$, eq. (25) becomes

$$
\left(\partial_{t}^{2}+\omega_{p}^{2}\right) \phi_{\mathbf{p}}=g_{n} \partial_{t} \int d k \Psi^{o}(\mathbf{p}, k, t)-g_{n} \partial_{t} \int d t^{\prime} \int d k R^{o}\left(t, t^{\prime} ; k, p\right) \partial_{t^{\prime}}\left(g_{n} \phi_{\mathbf{p}}\left(t^{\prime}\right)\right)
$$

where $g_{n} \equiv g \Lambda_{L V}\left(p / \Lambda_{L V}\right)^{n+1}$. On the other hand, for each 3-momentum $\mathbf{p}, \boldsymbol{\Psi}=\int d k \Psi(k)$ is a massless 2-dimensional free field. In Fourier components, its retarded Green function is given by $R^{o}(\omega, k)=1 /\left(-(\omega+i \epsilon)^{2}+\left(\pi \Lambda_{L V} k\right)^{2}\right)$. Hence $\mathbf{R}^{o}\left(t, t^{\prime}\right)$ obeys

$$
\partial_{t} \mathbf{R}^{o}\left(t, t^{\prime}\right) \equiv \partial_{t} \int_{-\infty}^{\infty} \frac{d \omega}{2 \pi} \int_{-\infty}^{\infty} d k R^{o}(\omega, k) e^{-i \omega\left(t-t^{\prime}\right)}=\frac{\delta\left(t-t^{\prime}\right)}{\Lambda_{L V}}
$$

which is the required property to simplify eq. (36).

When $g$ is constant, the retarded Green function of $\phi$ associated to eq. (36) obeys the following local equation

$$
\left[\partial_{t}^{2}+\frac{g_{n}^{2}}{\Lambda_{L V}} \partial_{t}+\omega_{p}^{2}\right] G_{r}\left(t, t^{\prime}, p\right)=\delta\left(t-t^{\prime}\right)
$$


To make contact with Appendix A and B, let us rewrite this equation in Fourier transform:

$$
\left[-\omega^{2}-i g^{2} \frac{\omega}{\Lambda_{L V}} p^{2}\left(\frac{p}{\Lambda_{L V}}\right)^{2 n}+\omega_{p}^{2}\right] G_{r}(\omega, p)=1 .
$$

We thus see that $\operatorname{Re} \Sigma_{r}=0$ and that $\operatorname{Im} \Sigma_{r}$ is (exactly) given by $g^{2}$ times that of eq. (85). Thus, even though we have chosen a simple form for $\mathbf{R}^{o}\left(t, t^{\prime}\right)$, the above action delivers the $n$ dissipative behaviors of Appendix B by choosing the appropriate power of $p / \Lambda_{L V}$ in $S_{\phi \Psi}$.

When $g$ and $\omega_{p}^{2}$ are arbitrary time-dependent functions, the Fourier analysis looses its power. However, in time dependent settings, our $G_{r}$ still obeys a local equation:

$$
\left[\partial_{t}^{2}+2 \tilde{\gamma}_{n} \partial_{t}+\left[\omega_{p}^{2}+\partial_{t} \tilde{\gamma}_{n}\right]\right] G_{r}\left(t, t^{\prime}\right)=\delta\left(t-t^{\prime}\right) .
$$

where the $n$-th decay rate $\tilde{\gamma}_{n}(t)=g^{2}(t) \gamma_{n}$ is now a definite time dependent function.

\subsection{Covariant description}

We now provide the covariantized expression of the action $S_{T}=\int d^{3} p S_{T}(\mathbf{p})$, where $S_{T}(\mathbf{p})$ is given in eq. (35). This expression will then be used to define our theory in curved backgrounds, 2

Two steps should be done. We need to go from $\mathbf{p}$ considerations to a local description, and express the various actions in terms of the unit vector field $l^{\mu}$ and the spatial metric $\perp^{\mu \nu}$. Both are straightforward and, in arbitrary coordinates, the action reads

$$
\begin{aligned}
S_{T}= & -\frac{1}{2} \int d^{4} x \sqrt{-g} g^{\mu \nu} \partial_{\mu} \phi \partial_{\nu} \phi \\
& \left.+\frac{1}{2} \int d^{4} x \sqrt{-g} \int d k\left[\left(l^{\mu} l^{\nu}-c_{\Psi}^{2} \perp^{\mu \nu}\right) \partial_{\mu} \Psi(k) \partial_{\nu} \Psi(k)-\left(\pi \Lambda_{L V} k\right)^{2} \Psi^{2}(k)\right)\right] \\
& +g \Lambda_{L V} \int d^{4} x \sqrt{-g}\left(\left(\frac{\Delta}{\Lambda_{L V}^{2}}\right)^{(n+1) / 2} \phi\right) l^{\mu} \partial_{\mu} \int d k \Psi(k),
\end{aligned}
$$

where the symbol $\Delta$ is the Laplacian on the three surfaces orthogonal to $l^{\mu}$.

We have slightly generalized the action of eq. (35) by subtracting $c_{\Psi}^{2} \perp^{\mu \nu}$ to the kinetic term of $\Psi$, where $c_{\Psi}^{2} \ll 1$. With this new term, the $\Psi(k)$ are now massive fields which propagate with a velocity whose square is bounded by $c_{\Psi}^{2}$. In addition they now possess a well defined energy-momentum tensor which can be obtained by varying their action with respect to $g^{\mu \nu}$. To obtain the simplified expressions we have used (and shall still use), the (regular) limit $c_{\Psi}^{2} \rightarrow 0$ should be taken.

\footnotetext{
${ }^{2}$ This procedure perhaps requires further explanation since $\Psi$ is not a fundamental field but "nothing more than a convenient parameterization of some environment degrees of freedom." First, $\Psi$ has not been introduced to parameterize the dissipative effects arising from any theory, but only those from theories obeying the Equivalence Principle, see 9] for a prototype. Second, from the point of view of cond-mat physics, it could a priori seem inappropriate to proceed to a covariantization, since in most situations (heat bath) there is a preferred frame which is globally defined. However, when the system is non-homogeneous (e.g. a fluid characterized by a nonhomogeneous flow), low energy fluctuations effectively live in a curved geometry[28]. Moreover, in this case, short distance effects, i.e. dispersive (or dissipative) effects, are covariantly described when using this metric because they arise locally [3, 11. Hence the covariantization is both a necessary step to implement the EP in our settings and a property that emerges in cond-mat physics.
} 
In this limit, the (free) retarded Green function of the $\boldsymbol{\Psi}$ field obeys a particularly simple equation when expressed in space-time coordinates:

$$
l^{\mu} \frac{\partial}{\partial x^{\mu}} \int d k R^{o}(x, y ; k)=l^{\mu} \frac{\partial}{\partial x^{\mu}} \mathbf{R}^{o}(x, y)=\frac{1}{\Lambda_{L V}} \frac{\delta^{4}\left(x^{\mu}-y^{\mu}\right)}{\sqrt{-g}} .
$$

On the r.h.s, one finds the delta function with respect to the invariant measure $d^{4} x \sqrt{-g}$. This equation is nothing by the covariantized and "localized" version of eq. (37). Its physical meaning is clear. In our model, the back-reaction of $\phi(x)$ onto itself through $\boldsymbol{\Psi}$ is local.

In spite of this, the equations of motions do not have a particularly simple form. Using the condensed notion $\Psi=\int d k \Psi$, one gets

$$
\frac{1}{\sqrt{-g}} \partial_{\mu} \sqrt{-g} g^{\mu \nu} \partial_{\nu} \phi(x)=g \Lambda_{L V} \frac{1}{\sqrt{-g}}\left(\frac{\Delta}{\Lambda_{L V}^{2}}\right)^{\frac{n+1}{2}} \sqrt{-g} l^{\mu} \partial_{\mu} \Psi(x),
$$

where the interacting $\mathbf{\Psi}(x)$ field is

$$
\boldsymbol{\Psi}(x)=\boldsymbol{\Psi}^{o}(x)-g \Lambda_{L V} \int d^{4} y \sqrt{-g} \mathbf{R}^{o}(x, y)\left[\frac{1}{\sqrt{-g}} \partial_{\mu}\left(l^{\mu} \sqrt{-g}\left(\frac{\Delta}{\Lambda_{L V}^{2}}\right)^{\frac{n+1}{2}} \phi(y)\right)\right] .
$$

When inserting eq. (44) in eq. (43), using eq. (42), one verifies that the dissipative term is local and first order in $l^{\mu} \partial_{\mu}$. Therefore, as expected, dissipation occurs along the preferred direction specified by the vector field $l^{\mu}$.

\subsection{Dissipative effects in curved background geometries}

To define a dissipative QFT in an arbitrary curved geometry, one needs some principles. From a physical point of view, we adopt the Equivalence Principle, or better what can be considered as its generalization in the presence of the unit time-like vector field $l^{\mu}$. We are in fact dealing with two (set of) dynamical fields, the $\phi$ field we probe, and the $\boldsymbol{\Psi}$ field we do not; but also with two background fields $g_{\mu \nu}$ and $l^{\mu}$. The Generalized Equivalence Principle means that the action densities of the dynamical fields be given by scalar functions (under general coordinate transformations) which coincide to those one had in Minkowski space time for a homogeneous and static $l^{\mu}$ field, i.e. those of eq. (41).

However, the densities are not completely fixed by the GEP. In this we recover what was obtained with the EP: For a scalar field in a curved geometry, there was always the possibility of considering a non-minimal coupling to gravity by adding to the Lagrangian a term proportional to $R \phi^{2}$. In the present case, the ambiguity is larger because $l^{\mu}$ defines new scalars, the first of which is the expansion $\Theta=\nabla_{\mu} l^{\mu}$, where $\nabla_{\mu}$ is the covariant derivative with respect to $g_{\mu \nu}$. The ambiguity can only be resolved by adopting some additional principle, such as the principle of minimal couplings which forbids adding densities containing these scalars.

Rather than adopting it, we shall choose the non-minimal coupling so as to keep eq. (42), i.e. so that the locality of the back-reaction effects of $\phi$ through $\Psi$ be preserved. This choice maintains the simplicity of the equations of motion in curved backgrounds, but is by no means necessary. Starting from eq. (41), the locality is preserved by replacing in $S_{\Psi}$ and $S_{\Psi \phi}$

$$
l^{\mu} \partial_{\mu} \Psi_{k} \rightarrow \mathcal{D}_{l} \Psi_{k} \equiv l^{\mu} \partial_{\mu} \Psi_{k}+\frac{\Psi_{k}}{2} \Theta=\frac{1}{2}\left(l^{\mu} \nabla_{\mu} \Psi_{k}+\nabla_{\mu}\left[l^{\mu} \Psi_{k}\right]\right)
$$


To simplify the forthcoming equations, we use the fact that one can always work in "preferred" coordinate systems in which the shift $l^{i}$ vanishes and in which the preferred time is such that $l_{0}=1$. (We assume that the set of orbits of $l^{\mu}$ is complete and without caustic. In this case, every point of the manifold is reached by one orbit.) In these coordinate systems, it is useful to work with rescaled fields $\Psi^{r} \equiv(-g)^{1 / 4} \Psi$ because the above equation simplifies

$$
\mathcal{D}_{l} \Psi_{k}=(-g)^{-1 / 4} l^{\mu} \partial_{\mu}\left((-g)^{1 / 4} \Psi_{k}\right)=(-g)^{-1 / 4} l^{\mu} \partial_{\mu} \Psi_{k}^{r},
$$

since $\Theta=(-g)^{-1 / 2} \partial_{\mu}\left[(-g)^{1 / 2} l^{\mu}\right]$. Notice also that there exists a subclass of background fields $(g, l)$, for which one can find coordinate systems such that both the shift $l^{i}$ and $g^{o i}$ vanish. In these comoving coordinate systems, the above equations further simplify since only the spatial part of the metric matters because $-g=h_{c}$ where $h \equiv \operatorname{det}\left(\perp_{i j}\right)$. 3

Having chosen this non-minimal coupling, one verifies that the kinetic term of the rescaled fields $\Psi_{r}$ is insensitive to the "curvature" of both $g_{\mu \nu}$ and $l^{\mu}$ (when the limit $c_{\Psi}^{2} \rightarrow 0$ is taken). Moreover the differential operator which acts on the retarded Green function of $\Psi_{r}$ in the equation of motion of $\phi$, see eqs. (43, 44), is also "flat" thereby guaranteeing that the modified version eq. (42) still applies, that is

$$
\begin{aligned}
\mathcal{D}_{l} \mathbf{R}^{o}(x, y) & =(-g(x))^{-1 / 4} l^{\mu} \frac{\partial}{\partial x^{\mu}}\left(\mathbf{R}_{r}^{o}(x, y)\right)(-g(y))^{-1 / 4} \\
& =\frac{1}{\Lambda_{L V}} \frac{\delta^{4}\left(x^{\mu}-y^{\mu}\right)}{\sqrt{-g}}
\end{aligned}
$$

where $\mathbf{R}_{r}^{o}(x, y)$ is the retarded Green function of the rescaled field $\Psi_{r}$. It obeys (in preferred coordinate systems) $\partial_{t} \mathbf{R}_{r}^{o}=\delta^{4} / \Lambda_{L V}$, and "defines" the retarded Green function $\mathbf{R}^{o}=$ $(-g)^{1 / 4} \mathbf{R}_{r}^{o}(-g)^{1 / 4}$ which is a bi-scalar. Hence the equations of motion in an arbitrary background "tensor-vector metric" specified by the couple $\left(g_{\mu \nu}, l^{\mu}\right)$, are given by eqs. (433, 44) with the substitution of eq. (45).

Several remarks should be made. First, from the simplified equation $\partial_{t} \mathbf{R}_{r}^{o}=\delta^{4} / \Lambda_{L V}$ it might seem that the background tensor metric $g_{\mu \nu}$ plays no role. This is not true, since it is used to normalize the field $l^{\mu}$ at every point.

Second, in the limit $c_{\Psi}^{2} \rightarrow 0$, the (rescaled) $\Psi_{k}$ fields define a new kind of field. They propagate in an effective space-time given by the time development of the 3 -dimensional set of orbits of the $l^{\mu}$ field. Indeed, at fixed $k, \Psi_{k}(x)$ can be decomposed in non-interacting local field-oscillators, each of them evolving separately along its orbit. This situation is similar to the long wave length (gradient-free) expression of [32]. In the absence of $l^{\mu}$, the geometry must be (nearly) homogenous for the action to posses this decomposition. However, when $l^{\mu}$ is given,

\footnotetext{
${ }^{3}$ It is clear that this is the case when $l^{\mu}$ coincides with the cosmological frame and when one uses comoving coordinates $d s^{2}=-d t^{2}+a^{2} d x^{2}$ since $l^{\mu}=(1,0)$. However, in certain cases one should search for the "comoving" coordinate system. To illustrate this point, consider the former situation in Lemaitre coordinates $X=a x$. In this case one has $d s^{2}=-d t^{2}+(d X-V d t)^{2}$, where the velocity is $V=H X$. The spatial sections are now the Euclidean space with $h=1$, and the (contravariant) components of the unit vector field are $l^{\mu}=1, V$. To compute $h_{c}$ one should solve the equation of motion of comoving (free falling) observers $d X-V d t=0$, and use the initial position as new coordinates. This procedure is explicitely done in [12] when starting with Painlevé-Gullstrand coordinates to describe the black hole metric and using a freely falling frame.
} 
one can identify, even in non-homogeneous metrics, each space-time point in an invariant way by the spatial position of the corresponding "preferred" orbit at some time, and the proper time along the orbit (as long as $l^{\mu}$ has no caustic). We can thus build covariant actions exploiting this possibility and consider fields composed of a dense set of local oscillators at rest with respect to $l^{\mu}$. The fields $\Psi_{k}$ we use belong to this class of fields.

\section{Dissipative effects in cosmology}

\subsection{The action}

Our aim is to describe dissipative effects in an expanding homogeneous universe when the vector field $l^{\mu}$ is aligned along the cosmological frame, when the dissipative effects are known in Minkowski space, and when implementing the Equivalence Principle.

In this case, to get the action we simply consider eq. (41) (with the curved metric modifications discussed in the former subsection) in a FLRW metric. Using comoving coordinates,

$$
d s^{2}=-d t^{2}+a^{2}(t) d \mathbf{x}^{2},
$$

the components of $l^{\mu}$ are $(1, \mathbf{0})$. To simplify the notations, we use the conformal time $d \eta=d t / a$ and work with the rescaled fields $\phi_{r}=a \phi$ and $\Psi_{r}=h_{c}^{1 / 2} \Psi=a^{3 / 2} \Psi$. Dropping these $r$ indices, working in Fourier transform with respect to $\mathbf{x}$, the non-minimally coupled action associated with the replacement of eq. (45) is

$$
\begin{aligned}
S_{T}^{(n)}(\mathbf{p})= & \frac{1}{2} \int d \eta \phi_{\mathbf{p}}^{*}\left(-\partial_{\eta}^{2}-\omega_{p}^{2}(\eta)\right) \phi_{\mathbf{p}} \\
& +\frac{1}{2} \int d t \int d k \Psi^{*}(\mathbf{p}, k)\left(-\partial_{t}^{2}-\left(\pi \Lambda_{L V} k\right)^{2}\right) \Psi(\mathbf{p}, k) \\
& +\int d \eta g_{n}(\eta) \phi_{\mathbf{p}} \partial_{\eta} \int d k \Psi^{*}(\mathbf{p}, k) .
\end{aligned}
$$

The conformal frequency $\omega_{p}^{2}(\eta)=p^{2}-\partial_{\eta}^{2} a / a$ is that of a rescaled minimal coupled massless field. In this expression, as everywhere in this Section, $p$ is now the conformal (dimensionless and constant) wave vector. The time dependent coupling coefficient is

$$
g_{n} \equiv g a^{1 / 2} \Lambda_{L V}\left(p / a \Lambda_{L V}\right)^{n+1} .
$$

Its dependence in $a$ (given that of $p$ ) follows from having implemented the Generalized Equivalence Principle (GEP) which determines the powers of $a$ for each term in the action. As we shall see, this relative power guarantees that all $\phi_{p}$ will be damped at a fixed and common proper scale (in the adiabatic approximation). Had we started with an action of the type (49) without relying on the GEP, the dependence in $a$ would have been arbitrary, and the proper scale at which modes would have been damped would have run as well.

In the same vein, one sees that the proper frequency of the $\Psi$ fields stays constant. This follows from the GEP but also from our choice of non-minimal couplings. In this paper, we want 
indeed to analyse the phenomenology of dissipative effects when the new degrees of freedom are and stay in their ground state. One could have chosen more complicated models in which $\Psi$ is parametrically excited. This would be the case when introducing a non zero velocity $c_{\Psi}$, and with minimally coupling (by dropping $\Theta$ on the rhs of $\equiv$ in eq. (45)). However, when $\Psi$ is taken massive (i.e. by restricting the $k$ range to $|k|>1$ ), or discretizing $k$ as it would be the case for Kaluza-Klein modes, and if $\Lambda_{L V} \gg H$, the phenomenology of all these models coincide since the amplitude for parametric excitations will be exponentially damped. In this regime there is thus no gain in studying more complicated actions than that given in eq. (49).

\subsection{Equation of motion}

Using the results of the subsection 2.5, the equation of motion of Heisenberg operator $\phi_{\mathbf{p}}$ is

$$
\left(\partial_{\eta}^{2}+2 \gamma_{n} \partial_{\eta}+\left(\omega_{p}^{2}(\eta)+\partial_{\eta} \gamma_{n}\right)\right) \phi_{\mathbf{p}}=g_{n} \partial_{\eta} \Psi^{o}(\mathbf{p})
$$

where the decay rate in conformal time is,

$$
\gamma_{n}(\eta)=\frac{g_{n}^{2}}{2 \Lambda_{L V}}=\frac{1}{2}\left(a \Lambda_{L V}\right)\left(\frac{p}{a \Lambda_{L V}}\right)^{2 n+2} .
$$

It is dimensionless, as it should be. It should be compared with the comoving frequency $p$ to get the relative strength of dissipation. One obtains

$$
\frac{\gamma_{n}(\eta)}{p}=\frac{1}{2}\left(\frac{p}{a \Lambda_{L V}}\right)^{2 n+1}=\frac{1}{2}\left(\frac{p_{p h y s}}{\Lambda_{L V}}\right)^{2 n+1} .
$$

In the last equality we have re-introduced the proper momentum $p_{\text {phys }}=p / a$. With this equation we verify that, at any time in an expanding universe and for every mode $\phi_{p}$, the relative strength of dissipation is simply that of Minkowski space evaluated at the corresponding energy scale, see eq. (86). This directly results from having implemented the GEP.

Notice however that the equation of motion in an expanding universe contains a frequency shift

$$
\frac{g_{n}^{2}}{\Lambda_{L V}} \partial_{\eta}\left(\ln g_{n}\right)=p^{2}\left(\frac{a H}{p}\right)\left(\frac{p}{a \Lambda_{L V}}\right)^{2 n+1}
$$

We have factorized out the unperturbed frequency square to get the relative value of the shift. It vanishes both when dissipation is negligible and when $a H / p \ll 1$, i.e. when the expansion rate $H$ is negligible with respect to the proper momentum. From this expression we can already conclude that it cannot play any role when the two relevant scales $H$ and $\Lambda_{L V}$ are well separated, i.e. when

$$
\sigma \equiv \frac{H}{\Lambda_{L V}} \ll 1
$$

Indeed when the physical momentum is high and of the order of $\Lambda_{L V}$, the relative frequency shift proportional to $\sigma$, and when the physical momentum is of the order of $H$ (at horizon exit, see Figure 1), it is proportional to $\sigma^{2 n+1}$.

In quantum settings however, it is not sufficient that the equation of motion possesses its Minkowskian form because the state of the system, and therefore the observables, might be affected by the combined effect of dissipative effects and the expansion rate. 


\subsection{Power Spectrum}

Let us consider the power spectrum of a scalar massless test field (which is the relevant case for gravitational waves and density perturbations) both in the standard free field settings and in the presence of dissipative effects.

\subsubsection{Free settings}

The equation of motion is the same as in eq. (10) with

$$
\omega_{p}^{2}=p^{2}-\frac{2}{\eta^{2}} \text {. }
$$

For simplicity we consider the inflationary background to be de Sitter where $a(\eta)=-1 / H \eta$ and $\eta<0$. At the onset of inflation, when $p_{\text {phys }} / H=p / a_{i n} H=p\left(-\eta_{\text {in }}\right) \rightarrow \infty$, the positive frequency modes are

$$
\phi_{\mathbf{p}}^{i n}=\frac{1}{(2 p)^{1 / 2}}\left(1-\frac{i}{p \eta}\right) e^{-i p \eta}
$$

When $p|\eta|=1$, the physical wave length $\lambda=a / p$ becomes larger than the Hubble radius. Near that "horizon-exit", $\omega_{p}^{2}$ flips sign, the mode stops oscillating and starts to grow like $a$. At late time with respect to horizon exit, when $|p \eta| \ll 1$, this implies that the power spectrum becomes a constant, as we now recall.

When inflation lasts long enough, i.e. when the number of extra e-folds obeys $N_{\text {extra }} \gg 1$, see eq. (1), all observable modes $\phi_{\mathbf{p}}$ are in their ground state at the onset of inflation (simply because this is the only state compatible with inflation [27]). In this case, the anti-commutator of the free field, see eq. (32), when evaluated at equal time $\eta$ is simply given by

$$
G_{a}^{\text {free }}(\eta, p)=\left|\phi_{\mathbf{p}}^{i n}\right|^{2}=\frac{1}{(2 p)}\left(1+\frac{1}{(p \eta)^{2}}\right) .
$$

Then the power spectrum of the physical (un-rescaled) field given by

$$
P_{p}^{\text {free }}(\eta)=\frac{p^{3}}{2 \pi^{2}} \frac{G_{a}^{\text {free }}(\eta, p)}{a^{2}(\eta)}=\left(\frac{H_{p}^{2}}{2 \pi}\right)^{2}\left(1+(p \eta)^{2}\right),
$$

becomes constant after horizon exit. We have added a $p$ subscript to $H$ because in slow roll inflation, the relevant value of $H$ for the $p$-mode is that evaluated at horizon exit, i.e. $H_{p}=H\left(t_{p}\right)$, where $t_{p}$ is given by $p / a=H$. The above equation shows that $P_{p}$ acquires some scale dependence only through $H_{p}$. Similarly the deviations from this standard behavior stemming from some UV modification of the theory will also depend on $p$ through $H_{p}$ (and its derivatives). For explicit examples, we refer to [30] where the modifications of the spectrum stem from the fact that $p \eta_{i n}$ is taken large but finite, and also to [31] wherein the UV cutoff is endowed with a finite width. This last case bears many similarities with the dissipative settings we now study. 


\subsubsection{Dissipative settings}

In the presence of dissipation, the expression for $G_{a}$ radically differs from the above.

At the level of the Heisenberg operator, when dissipative effects grow with the energy (as we suppose it is the case), the decaying solution of eq. (51) is completely erased (unless one fine-tunes $N_{\text {extra }}$, see eq. (11), so as to keep a residual amplitude). That is, in inflation the mode operator is entirely given by its driven term, the second term in eq. (26). Then the power spectrum is also purely driven and given by the second term of eq. (33):

$$
G_{a}^{d r i v e n}(\eta, p)=\int d \eta_{1} \int d \eta_{2} G_{r}\left(\eta, \eta_{1}, p\right) G_{r}\left(\eta, \eta_{2}, p\right) \mathbf{N}\left(\eta_{1}, \eta_{2}, p\right)
$$

where $G_{r}$ is the retarded Green function, solution of eq. (51) with $\delta\left(\eta-\eta_{1}\right)$ as a source, and where the kernel $\mathbf{N}$ is the anti-commutator of $g_{n} l^{\mu} \partial_{\mu} \boldsymbol{\Psi}_{p}$, the source of eq. (51).

Eq. (60) tells us that only the state of the environment matters. In other words, because of the strong dissipation at early times, the power spectrum is independent of the initial state of $\phi$, what ever it was. In spite of this, when eq. (55) is satisfied, and when the environment is in its ground state, the predictions are unchanged, i.e. the power spectrum obtained from $G_{a}^{d r i v e n}$ coincides with that obtained with $G_{a}^{f r e e}$. To show this let us study $G_{r}$ and $\mathbf{N}$.

\subsubsection{Dissipative Green functions and noise kernel}

The retarded Green function is of the form

$$
G_{r}\left(\eta, \eta_{0}, p\right)=\theta\left(\eta-\eta_{0}\right) e^{-\int_{\eta_{0}}^{\eta} d \eta^{\prime} \gamma\left(\eta^{\prime}\right)} \times 2 \operatorname{Im}\left(\tilde{\phi}_{p}^{*}(\eta) \tilde{\phi}_{p}\left(\eta_{0}\right)\right)
$$

where (in the under-damped regime) the modes $\tilde{\phi}_{p}$ are unit Wronskian positive frequency solutions of eq. (56) with a frequency square given by

$$
\left(\omega_{p}^{e f f}\right)^{2}=\omega_{p}^{2}-\gamma_{n}^{2}=p^{2}\left(1-\frac{2}{p^{2} \eta^{2}}-\frac{\gamma_{n}^{2}}{p^{2}}\right)
$$

In this, we obtain a time-dependent version of the stationary case, see eq. (87). With the second expression, we verify that when eq. (55) is satisfied, the two corrections terms are never simultaneously relevant, which guarantees, as we shall discuss below, that dissipative effects can be studied in the quasi-stationary approximation. Notice also that the frequency shift $\partial_{\eta} \gamma$ present is eq. (54) drops out from $\omega^{\text {eff }}$.

Eq. (61) implies that

$$
G_{r}\left(\eta, \eta_{0}, p\right) \rightarrow 0 \quad \text { when } \quad \eta_{0} \rightarrow \eta_{p}^{\Lambda}
$$

where $\eta_{p}^{\Lambda}$ is the " $\Lambda_{L V}$-exit" time of the $p$-mode defined by $p / a\left(\eta_{p}^{\Lambda}\right)=\Lambda_{L V}$, or $\gamma / p=1 / 2$, see eq. (53) and the Figure. 


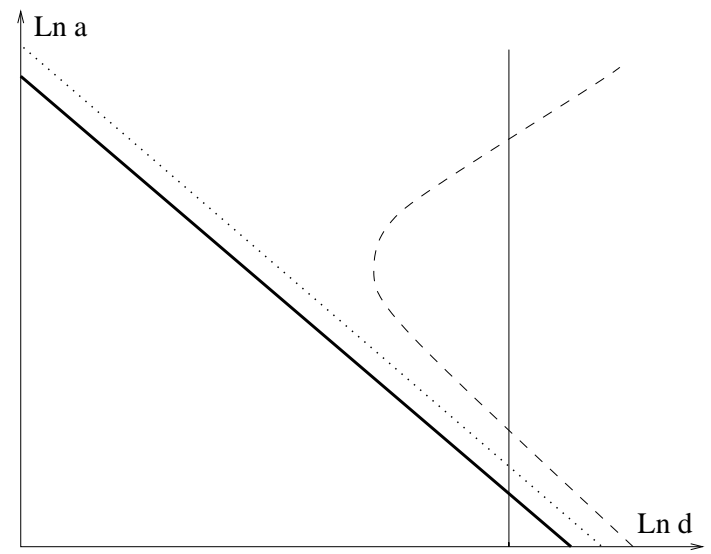

Figure caption. We have represented in a log-log plot and by a dashed line the evolution of $d_{H}=R_{H} / a$, the comoving Hubble radius, as a function of a, both during inflation $\left(d_{H} \propto 1 / a\right)$ and during the radiation era $\left(d_{H} \propto a\right)$. We have represented by a thick line the trajectory of the cutoff length $d_{\Lambda}=1 / a \Lambda_{L V}$ of a fixed proper scale which obeys $1 / \Lambda_{L V} \ll R_{H}$ during inflation. The dotted line corresponds to an intermediate fixed proper length $\lambda$ which obeys $1 / \Lambda_{L V} \ll \lambda \ll R_{H}^{i n f l}$. The vertical line represents a fixed comoving scale $d_{p}=1 / p$. Below the cutoff length, all modes are over-damped. When a mode exits the cutoff length, it becomes under-damped and starts propagating. When it crosses the intermediate length $\lambda$, it behaves as a free mode, and it gets amplified only when exiting the Hubble radius. Adiabaticity, which is guaranteed by $1 / \Lambda_{L V} \ll R_{H}$, guarantees in turn that, near $\lambda$, modes are all born in the Bunch-Davies vacuum when the environment $\boldsymbol{\Psi}$ is in its ground state.

Eq. (63) guarantees that no quantum coherence is left between late time $\eta$, e.g. horizonexit, and what happened for times earlier than $\Lambda_{L V}$-exit. The physical implication of this is that, what ever transitions happened, what ever was the quantum state, no record is kept at late time 4 Having understood the structure of the retarded Green function, let us now briefly consider the commutator $G_{c}\left(\eta, \eta_{0}\right)$. It is given by

$$
\begin{aligned}
G_{c}\left(\eta, \eta_{0}, p\right) & =-i\left(G_{r}\left(\eta, \eta_{0}, p\right)-G_{r}\left(\eta_{0}, \eta, p\right)\right) \\
& =e^{-\left|\int_{\eta_{0}}^{\eta} d \eta^{\prime} \gamma\left(\eta^{\prime}\right)\right|} \times 2 i \operatorname{Im}\left(\tilde{\phi}_{p}(\eta) \tilde{\phi}_{p}^{*}\left(\eta_{0}\right)\right) .
\end{aligned}
$$

It possesses two noteworthy properties. First, because of the absolute value in the damping exponential term, it obeys two different local differential equations depending of the sign of $\eta-\eta_{0}$. When $\eta>\eta_{0}, G_{c}$ is an homogeneous solution of eq. (51), whereas when $\eta<\eta_{0}$, it obeys the equation wherein the sign of the dissipative term has been changed. These two differential

\footnotetext{
${ }^{4}$ This behavior has been described, drawn, and sometimes named anti-dissipation, see [5, 11, 7, 33, 34, 35, 14. In fact when tracing backwards in time vacuum configurations from the freely propagating regime down to the dissipative one, dissipation arises towards the past. When viewed as a function of $\eta_{0}, G_{r}\left(\eta, \eta_{0}\right)$ indeed increases to the future, thereby showing anti-dissipation. From eq. (61) we understand the origin of this quantum property (without classical counterpart). As a function of $\eta_{0}$, first, $G_{r}$ obeys eq. (51) with the "wrong" sign for the dissipative term, and second, the boundary condition is a final one and not an initial one, thereby explaining why when $\eta_{0} \rightarrow \eta$, the value of the current is "adjusted" to become exactly one.
} 
equations can be grouped into a single non-local equation. Second, because of this "quasi"-local properties, $G_{c}$ is independent of $\eta_{i n}$, the moment when $\phi$ was put in contact with $\Psi$.

These properties of $G_{c}$ follow from the fact that its source term, the $D$ kernel of eq. (29), is "ultra" local for the $\boldsymbol{\Psi}$ field of eq. (49):

$$
\mathbf{D}\left(\eta_{1}, \eta_{2}\right) \equiv\left[g_{n} \partial_{\eta_{1}} \boldsymbol{\Psi}\left(\eta_{1}\right), g_{n} \partial_{\eta_{2}} \boldsymbol{\Psi}\left(\eta_{2}\right)\right]=g_{n}\left(\eta_{1}\right) \frac{i \partial_{\eta_{2}} \delta\left(\eta_{1}-\eta_{2}\right)}{\Lambda_{L V}} g_{n}\left(\eta_{2}\right)
$$

This should be contrasted with the kernel $\mathbf{N}$, the anti-commutator, which is non local. Indeed, in the vacuum, it is given

$$
\mathbf{N}\left(\eta_{1}, \eta_{2}\right)=g_{n}\left(\eta_{1}\right) \frac{-a\left(\eta_{1}\right) a\left(\eta_{2}\right)}{\pi \Lambda_{L V}\left(t_{1}-t_{2}\right)^{2}} g_{n}\left(\eta_{2}\right)
$$

where $1 /\left(t_{1}-t_{2}\right)^{2}$ should be understood as the derivative of the principal part of $1 /\left(t_{1}-t_{2}\right)$. One gets a simple expression in terms of the proper time $t$ because the proper frequency of $\Psi_{k}$ is constant in our model (49). We also remind the reader that it is only in the high temperature limit that $N$ would become proportional to $\delta\left(t_{1}-t_{2}\right)$.

In this paper we shall compute the power spectrum when the $\Psi_{k}$ are all in their ground state. The reason for this choice is as follows: As pointed out in the footnote 2, the $\Psi_{k}$ fields should be conceived as parametrizing some degrees of freedom in a fundamental theory obeying the Equivalence Principle. In these theories, when inflation lasts long enough, all relevant degrees will be in their ground state, as is the case when dealing with free modes, see eq. (158). We see no reason why dissipation could possibly invalidate this conclusion.

When the $\Psi_{k}$ are all in their ground state, the kernel $\mathbf{N}$ is non-local. Therefore the anticommutator $G_{a}$ obeys a non-local equation: eq. (51) with $\mathbf{N}$ as the source. The physical meaning is that the environment is driving $\phi_{p}$ in an non-instantaneous way (this is unavoidable in the vacuum since only one sign of the frequency is present). The consequence is that the value of power spectrum depends (to a certain extend) on the history of the combined evolution of $\phi+\Psi$. The mathematical implication is that an exact calculation of $G_{a}$ is effectively impossible.

Nevertheless when $H / \Lambda_{L V} \ll 1$, these non local effects give only but sub-leading (nonadiabatic) corrections, because the evolution of $\phi+\Psi$ consists in a parametric (adiabatic) succession of stationary states ordered by the scale factor $a$.

\subsubsection{Scale separation and adiabatic evolution}

Given the importance of this result, we shall spend some time to explain its root and its validity. We proceed in two steps. We first show that adiabaticity is sufficient for obtaining the standard power spectrum, and then show that scale separation, eq. (55), is sufficient to guarantee adiabaticity, thereby generalysing the results of [8].

Adiabaticity means that the evolution proceeds slow enough for inducing no (non-adiabatic) transition (in molecular physics, they are called Landau-Zener transitions, in the present cosmological context they correspond to pair creation of $\phi$ quanta). When this is the case, the observables take, at any time, the value they have in the corresponding stationary situation. 
As of retarded Green function and the commutator, this principle does not bring any new information because in the WKB approximation, they are local functions since these 2pt functions obey local equations. For the anti-commutator, adiabaticity guarantees that when $\eta$ and $\eta^{\prime}$ are close (in the sense that $1-a(\eta) / a\left(\eta^{\prime}\right) \ll 1$ ), its value is well approximated by

$$
G_{a}^{\text {driven }}\left(\eta, \eta^{\prime}, p\right) \simeq G_{a}^{\text {statio }}\left(\eta-\eta^{\prime} ; \omega_{p}(a), g_{p}(a)\right)=\int \frac{d \omega}{2 \pi} e^{i \omega\left(\eta-\eta^{\prime}\right)} G_{a}\left(\omega ; \omega_{p}(a), g_{p}(a)\right),
$$

where $G_{a}\left(\omega ; \omega_{p}(a), g_{p}(a)\right)$ is the Fourier component calculated with the values of the frequency $\omega_{p}(a)$ and the coupling $g_{p}(a)$ both evaluated with $a=a(\eta)$. In Appendix A, these Fourier components have been algebraically solved for all frequencies and all couplings, see eq. (811). Moreover, since by hypothesis, we are in the vacuum, eq. (79) also applies. In other words, the value of $G_{a}$ follows from that of the commutator $G_{c}$. This is sufficient to guarantee that when the mode $\phi_{p}$ becomes free, i.e. much after $\Lambda_{L V}$-exit but before horizon-exit $\left(H \ll p / a \ll \Lambda_{L V}\right)$, eq. (84) applies. Thus, irrespectively of what was the coupling with the environment,

$$
G_{a}^{\text {driven }}(\eta, \eta, p) \rightarrow G_{a}^{\text {free }}(\eta, \eta, p)=\frac{1}{2 \omega_{p}(a)} .
$$

With this equation we reach our first conclusion: in the adiabatic approximation and when the environment is in its ground state, the modes $\phi_{p}$ are born in the usual in-vacuum (BunchDavies) as they become, one after the other, freely propagating after $\Lambda_{L V}$-exit, see the Figure.5

It now behooves to us to show that scale separation guarantees that eq. (67) provides a valid approximation for $G_{a}^{\text {driven }}$ before horizon-exit, i.e. when the second term in the parenthesis in eq. (62) can still be neglected. To this end, we provide an upper bound for the probability amplitude of getting a non-adiabatic transition in the under-damped regime, i.e. $H \ll p / a \leq \Lambda_{L V}$. This amplitude is governed by the relative frequency change

$$
\frac{\partial_{\eta} \omega_{p}^{e f f}}{\left(\omega_{p}^{e f f}\right)^{2}}=-\frac{\gamma_{n}^{2} \partial_{\eta} \ln \gamma_{n}}{\left(p^{2}-\gamma_{n}^{2}\right)^{3 / 2}}=(2 n+1) \frac{H \gamma_{\text {phys }}^{2}}{\left(p_{\text {phys }}^{2}-\gamma_{\text {phys }}^{2}\right)^{3 / 2}},
$$

where $p_{\text {phys }}=p / a$ and $\gamma_{p h y s}=\gamma / a$ are the physical (proper) momentum and decay rate. Therefore, going backwards in time from the free regime up to $p_{\text {phys }}^{2} \geq 4 \gamma_{\text {phys }}^{2}$ (i.e. $p_{\text {phys }} \leq \Lambda_{L V}$ ), the non-adiabatic parameter steadily grows but stays bounded by

$$
\frac{\partial_{\eta} \omega_{p}^{e f f}}{\left(\omega_{p}^{e f f}\right)^{2}}<3(2 n+1) \sigma\left(\frac{p_{p h y s}}{\Lambda_{L V}}\right)^{4 n+1}<3(2 n+1) \sigma \ll 1 .
$$

This guarantees that the amplitude for the system to jump out of the ground state is bounded by $\sigma$ (up to an overall factor which plays no role). There is no need to study the stability of the ground state in the transitory regime from under-damped to the over-damped modes because what ever transitions happened their residual impact at late time would be suppressed by a factor $e^{-\int \gamma d t} \simeq \exp (-1 / \sigma(2 n+1)) \ll 1$. This completes the proof that scale separation guarantees adiabaticity, in agreement with the conclusion reached in [9, 10].

${ }^{5}$ This behavior is in accord with the procedure of 29, 30, 31 wherein the initial state is imposed on modes when their proper momentum reaches a given value. This radically differs with the "initial slice" approach 36 . wherein the state is imposed at the same time on all modes, irrespectively of the value of their momentum. From what we see, the Equivalence Principle allows only the first to be dynamically realized. 


\subsubsection{Perspectives: Beyond the adiabatic approximation and scale crossing}

It is first interesting to point out that dissipative models are more "robust" than dispersive models in that the "driven" power spectrum determined by eq. (60) is well defined even when $H>\Lambda_{L V}$, contrary to the fact that most dispersive models make sense only when scale separation $\left(H \ll \Lambda_{L V}\right)$ is realized. Therefore, with dissipative models, one can study scale crossing, i.e. the crossing of $H_{p}$ through $\Lambda_{L V}$ from above to below during slow roll inflation.

The power spectrum has been studied in this regime when using a particular Brane World scenario in [10]. We agree with most of the conclusions but not with that reached after Eq. (35) according to which "towards the end of inflation, perturbations on the brane are the sum of two independent fields". We do not agree because in those settings as well the field operator is purely driven. Hence we do not see how to decompose it as a superposition of two commuting operators. Moreover, with the approximation used in [10], it seems that the ETC, eq. (11), is violated by a amount proportional to the reported change of the power spectrum.

In any case, the calculation of the modifications of the power spectrum introduced by dissipation is difficult because the non-local properties of eq. (60) cannot be neglected. We are not aware of any analytical treatment, and we are currently studying the modifications using numerical techniques 38].

Added note. Since the present paper was submitted, we have obtained several results [38]. In particular, the leading modification of the power spectrum with respect to the standard result in the regime $\sigma=H / \Lambda \ll 1$, i.e. the signature of UV dissipation, scales with a power of $\sigma$ which is equal to that of $P / \Lambda$ in $\gamma / p$, see eq. (53). In this respect, dissipative models behave like dispersive models where the leading modification scales with a power of $\sigma$ which is that of the first non-linear term in the dispersion relation, i.e. $2 n$ using the parameterization of eq. (22), see [39]. In the opposite regime, when $H>\Lambda$, no universal behavior is obtained.

\section{Conclusions}

In this paper we have obtained the following results.

First, we have provided a class of unitary models defined in Minkowski space which are characterized by the power of $p / \Lambda_{L V}$ which weighs the growth of dissipation in the preferred frame, see eq. (85). Unitarity is achieved by introducing a dense set of additional fields $\boldsymbol{\Psi}$ which induce dissipation through interactions with $\phi$. In Appendices A and B we have given a thorough analysis of the Green functions of these Gaussian models which cover the phenomenology of UV dissipative effects in Minkowski space at the level of 2 pt functions.

Second, amongst the various actions delivering the same stationary phenomenology, we have selected one which gives rise to a local differential equation for the retarded Green function of $\phi$, see eqs. (36, 37). Using the Equivalence Principle, we have extended this class of models to arbitrary curved backgrounds in subsection 2.7, thereby allowing to confront the trans-Planckian question of inflationary cosmology and black hole physics.

Third, we have applied our dissipative models to inflationary cosmology. At early times, hence at very high energy, the dissipative effects are so strong that all information about the initial state of the $\phi$ is erased, see eq. (63) . Nevertheless, when the UV scale $\Lambda_{L V}$ is much 
larger than the Hubble parameter, we have demonstrated that the standard expression of the power spectrum is found when the environment is in its ground state. The reason for this is the following: even though the field oscillator $\phi_{p}$ is purely driven by $\boldsymbol{\Psi}_{p}$, i.e. it is given by the second term eq. (26), as its proper momentum $p / a$ redshifts under $\Lambda_{L V}$, the composite operator behaves as if it were a free operator, see eqs. (31, 84), thereby guaranteeing eq. (68).

Let us also note that our models can be used for phenomenological purposes in the sense of [1], and that we are planning to apply them to study Hawking radiation in the presence of dissipation. We are presently completing the calculation of the power spectrum beyond the adiabatic approximation so as to determine the signatures of dissipation [38] and to compare them to those of dispersion [39]. Finally it would be interesting to compute the VEV of the stress-tensor of the $\boldsymbol{\Psi}$ fields in non-trivial backgrounds. This would allow to take into account the back-reaction on the cosmological metric engendered by the fields $\boldsymbol{\Psi}$ and $l^{\mu}$ [40, 41, 42].

\section{Acknowledgments.}

I am grateful to Dani Arteaga and Enric Verdaguer for common work allowing me to deepen my understanding of dissipative effects. I am also grateful to Julian Adamek, David Campo, Ted Jacobson, Jean Macher, Jens Niemeyer, and Valeri Rubakov for interesting discussions. I wish to thank the organizers of the workshops on "Micro and Macro structure of spacetime" held in Peyresq in June 2005, 2006, and 2007 where this work has been presented and discussed. This work has been supported by the Agence Nationale de la Recherche (projet 05-1-41810).

\section{Appendix A : Stationary states and vacuum 2 pt functions}

In this Appendix, we recall the (well-known) relationships between $G_{c}, G_{a}$ which always hold in stationary states. In these states, $G_{c}, G_{a}$ and the kernels $D, N$ are related to each other in a universal way, generally referred as a Fluctuation-Dissipation relation. We explain its origin and its physical implications in the present context. We start with the most basic object: the retarded Green function $G_{r}$.

\subsection{The retarded Green function}

The Fourier transform of eq. (25) gives

$$
\left(-\omega^{2}+\omega_{p}^{2}\right) \phi_{\mathbf{p}}(\omega)=\Sigma_{i} g_{i}(p) \Psi_{i}^{o}(\mathbf{p}, \omega)+\Sigma_{i} g_{i}^{2}(p) R_{i}^{o}(\omega ; p) \phi_{\mathbf{p}}(\omega)
$$

where

$$
\left.R_{i}^{o}(\omega ; p)=\left(-(\omega+i \epsilon)^{2}+\Omega_{i}^{2}(p)\right)\right)^{-1},
$$

is the Fourier transform (defined as in eq. (17)) of the retarded Green function of $\Psi_{i}$. As usual, its retarded character is enforced by the imaginary prescription of the two poles to lay in the lower half plane $(\epsilon>0)$. The solution of the above equation is

$$
\phi_{\mathbf{p}}(\omega)=\phi_{\mathbf{p}}^{d}(\omega)+G_{r}(\omega, p) \Sigma_{i} g_{i}(p) \Psi_{i}^{o}(\mathbf{p}, \omega),
$$


where the Fourier transform of the retarded Green function of $\phi$, the solution of eq. (27), always takes the form

$$
G_{r}(\omega, p)=\left(-(\omega+i \epsilon)^{2}+p^{2}+\Sigma_{r}(\omega, p)\right)^{-1} .
$$

All effects of the coupling to the $\Psi_{i}$ 's are thus encoded in the (retarded) self-energy $\Sigma_{r}(\omega, p)$. For Gaussian theories, it is algebraically given by

$$
\Sigma_{r}(\omega, p)=-\Sigma_{i} g_{i}^{2}(p) R_{i}^{o}(\omega, p) .
$$

The dissipative effects are governed by the imaginary part of $\Sigma_{r}(\omega, p)$. In the present case, one has

$$
2 \operatorname{Im} \Sigma_{r}(\omega, p)=-\Sigma_{i} g_{i}^{2}(p) G_{c, i}^{o}(\omega, p)=-D(\omega, p) .
$$

To get the first equality we have used the fact that in stationary states the retarded Green function and the commutator are related by $2 \operatorname{Im} G_{r}(\omega)=G_{c}(\omega)$ for all degrees of freedom, free or interacting. In the second equality, we have introduced $D(\omega)$, the Fourier transform of the kernel of eq. (30).

Several observations should be made here. First, from eq. (72), we obtain that $D(\omega)$ is proportional to $\Sigma_{i} g_{i}^{2} \delta\left(\omega-\Omega_{i}\right)$. Therefore there is no dissipation for lower frequencies than the lowest value of $\Omega_{i}$. This simply follows from energy conservation. Second, to obtain "true" dissipation, $D(\omega, p)$ should be a continuous function and not a sum of delta. This can only happen when the $\Psi_{i}$ form a dense ensemble. In Section 2.5, we shall thus replace the discrete sum on $i$ by an integral over a continuous variable, $k$. We shall not consider the discrete cases even though these could display interesting properties. Third, from a phenomenological point of view, only $D(\omega, p)$ matters. Hence we cannot disentangle the spectrum of the environment, which is given by $R_{i}^{o}(\omega, p)$, from the coupling strength $g_{i}^{2}(p)$. This is a good thing, because when working in time-dependent settings, we shall exploit this equivalence to chose the simplest model of $\Psi_{i}$ 's which gives the kernel $D(\omega, p)$ we want.

It is also worth noticing that the dispersive (real) effects are not directly related to $D$ (or $N)$. These are governed by the even part of $\Sigma_{r}(\omega, p)$ which is given by

$$
\operatorname{Re} \Sigma_{r}(\omega, p)=\int \frac{d \omega^{\prime}}{2 \pi} \frac{D\left(\omega^{\prime}, p\right)}{\omega-\omega^{\prime}}
$$

where the integral should be understood as a principal value. This integral relationship explains why one often founds that dispersive effects appear before dissipative effects (for increasing $\omega$ ). We also learn that the dispersive models studied in the literature violate the above relation since they assume $\operatorname{Re} \Sigma_{r} \neq 0$ and $\operatorname{Im} \Sigma_{r} \equiv 0$. Therefore these models are incoherent and cannot result from dynamical processes.

\subsection{Fluctuation-Dissipation relations and vacuum self-energy}

In this subsection, we derive the relationships between $G_{c}, G_{a}$ and $\Sigma_{F}$ which exist in the true (interacting) ground state. 
In interacting theories, the only stationary states are thermal states, see e.g. [37]. In these states, the Fourier transform of $D$ and $N$ are related by

$$
\begin{aligned}
N(\omega) & =D(\omega) \operatorname{coth}(\beta \omega / 2) \\
& =D(\omega) \operatorname{sign}(\omega)[2 n(|\omega|)+1] .
\end{aligned}
$$

In the second line, $n(\omega)$ is the Planck distribution. It gives the mean occupation number of $\Psi_{i}^{o}$ quanta as a function of the frequency (measured in the rest frame of the bath). The above relation directly follows from the fact that the individual commutators and anti-commutators of the free fields $\Psi_{i}^{o}$ obey this relation, as any free oscillator would do. It implies that the Fourier transform of $G_{c}$ and $G_{a}$ are also related by

$$
G_{a}(\omega)=G_{c}(\omega) \operatorname{sign}(\omega)[2 n(\omega)+1]
$$

It should be stressed that this equation is exact, i.e. non-perturbative, and valid for all theories, Gaussian or not. (It indeed directly follows from the cyclic properties of the trace defining $\left.G_{\beta}\left(t, t^{\prime}\right)=\operatorname{Tr}\left[e^{-\beta H_{T}} \phi(t) \phi\left(t^{\prime}\right)\right]\right)$.

For Gaussian models, there exists an alternative proof of eq. (79). It suffices to note that in steady states the decaying terms of eqs. (29) and (33) play no role, and that the Fourier transform of the driven terms are respectively given by

$$
\begin{aligned}
& G_{c}(\omega)=\left|G_{r}(\omega)\right|^{2} D(\omega), \\
& G_{a}(\omega)=\left|G_{r}(\omega)\right|^{2} N(\omega),
\end{aligned}
$$

since the Fourier transform of the retarded Green function obeys $G_{r}(\omega)=G_{r}^{*}(-\omega)$, see eq. (73). Irrespectively of the complexity of $G_{r}$, i.e. irrespectively of the functions $g_{i}(p), \Omega_{i}(p)$ and the set of the $\Psi_{i}$ fields, $G_{c}$ and $G_{a}$ are thus related to each other by the FD relation (79)).

These universal relations will be relevant for inflationary models wherein only the ground state contributes. In particular, they imply that in the true vacuum, i.e. when $n(\omega)=0, G_{c}$ and $G_{a}$ are exactly related by $G_{a}(\omega)=G_{c}(\omega) \operatorname{sign}(\omega)$. Hence the Wightman function

$$
G_{W}=\frac{1}{2}\left(G_{c}+G_{a}\right)=G_{c} \theta(\omega)
$$

is determined by the commutator and contains only positive frequency, as in the free vacuum. Equations (80, 81) also allow to compute the vacuum self-energy of the Feynman Green function. For Gaussian models it is given by

$$
2 \operatorname{Im} \Sigma_{F}(\omega, p)=-D(\omega, p) \operatorname{sign}(\omega) .
$$

With the last equality we recover the fact that in the vacuum, it is sufficient to consider Feynman Green functions. In non-vacuum states, and in non-stationary situations, this is no longer true, thereby justifying the use of the in - in machinery [20] (the Schwinger-Keldish formalism).

Before specializing to a specific class of models giving rise to dissipation at high frequency, we make a pause by asking the following important question: What should be known about 
the $\Psi_{i}$ fields to get eqs. (80, 81, 82) ? We have proven that it is sufficient for the $\Psi_{i}$ 's to be canonical fields, but is it necessary?

The answer is two fold. On one hand, the $\Psi_{i}$ cannot be stochastically fluctuating quantities (i.e. commuting variables) because this would lead to a violation of eq. (78) that would imply the violation of eq. (79) and the ETC eq. (31) 6 They cannot be either a combination of quantum and stochastic quantities because this would still lead to a violation of the ETC. Hence they must be built only from quantum (canonical) degrees of freedom.

On the other hand, the $\Psi_{i}$ 's can be composite operators, i.e. polynomials of some (unknown) canonical fields. Indeed, their commutators would still be all related to their anti-commutators by the FD relation eq. (78), and this even though they depend non-linearly on $n(\omega)$ in nonvacuum states. The difference with Gaussian models is that these non-linear operators posses non-vanishing higher order correlation functions. Hence, the self-energies $\Sigma_{r}, \Sigma_{F}$ will be series in powers of $g_{i}^{2}$, and not just a single quadratic term as in eq. (75, 83). Nevertheless these higher loops corrections preserve the validity of eq. (82) in the ground state, as well as that of eqs. (80, 81) in any thermal state, when properly understood, i.e. with $D$ now defined by $-2 \operatorname{Im} \Sigma_{r}$ (as the effective dissipation kernel), and $N$ related to it by the FD relation.

In brief, we have reached/recalled the following results. Firstly, the q-number combination $\Psi=\Sigma_{i} g_{i} \Psi_{i}$, the fluctuating source term of $\phi$, must obey the FD relation (78). This can either be postulated, or better, be viewed as resulting from the fact that $\boldsymbol{\Psi}$ is entirely made out of quantum degrees of freedom. Secondly, to lowest order term in $g$, the self-energy can be obtained by treating $\boldsymbol{\Psi}$ as a Gaussian variable, what ever its composition may be. Thirdly, when dealing with non-Gaussian theories, once having computed $\Sigma_{r}(\omega)$, the resulting equations for the 2pt functions have the same structure and the same meaning as in Gaussian theories, with $D$ replaced by $-2 \operatorname{Im} \Sigma_{r}$. Therefore, the phenomenology of two-point functions is entirely covered by Gaussian settings.

\subsection{The double limit: $g^{2} T \rightarrow \infty$ followed by $g^{2} \rightarrow 0$.}

To perform a phenomenological analysis of dissipation, we need to understand how the theory behaves in transitory regime from dissipative to free propagation. Similarly, to study primordial spectra in inflation or Hawking radiation, we also need to understand how free motion emerges as the proper frequency get red-shifted. It is therefore relevant to study the behavior of the two-point function in the following double limit.

One first takes $g^{2} T \rightarrow \infty$, where $T=t-t_{i n}, t_{i n}$ being the moment when the interactions are turn on, and $t$ the moment when one studies the field properties. This limit implies that the decaying term in eq. (26) plays no role. Therefore, near time $t$, the Heisenberg field $\phi(t)$ is a composite operator which only acts in the Hilbert space of $\boldsymbol{\Psi}$.

Secondly, one considers the "free" limit $g^{2} \rightarrow 0$ of that composite operator. One could naively conclude that $G_{c}$ and $G_{a}$ of eqs. (80, 81) would vanish since both $D$ and $N$ are

6 This constitutes the simplest proof that it is inconsistent to couple quantum variables to stochastic (or classical) ones. If one does so, the ETC of the dressed quantum variables will always be dissipated after a time of the order of $\gamma^{-1}$. One can therefore view the experimental evidences for the ETC of some degrees of freedom as a very strong indication that all dynamical variables in our world are quantum mechanical in nature. 
proportional to $g^{2}$. However, this is not the case, because the common prefactor, $\left|G_{r}\right|^{2}$, is singular in this second limit. In fact, one verifies that it scales in $1 / g^{2}$ in such a way that, in the (interacting) vacuum, one always recovers

$$
G_{W}(\omega)_{g^{2} \rightarrow 0}=\frac{1}{2 \omega_{p}} 2 \pi \delta\left(\omega-\omega_{p}\right) .
$$

This is the standard vacuum fluctuations of a free massless mode of momentum $p$.

Two important lessons have been reached. First we learned is that even though $\phi$ acts only on the $\boldsymbol{\Psi}$-Hilbert space, when $g^{2} \rightarrow 0$, it behaves as if it were a free mode possessing its own Hilbert space, with no reference to $\boldsymbol{\Psi}$-dynamics. Secondly, the quantum state in this would be Hilbert space is still exactly that of $\boldsymbol{\Psi}$. Therefore, in stationary situations, the only "souvenir" kept by the composite operator is the equilibrium distribution $n(\omega)$ inherited from its parents.

Let us now emphasize that the above limit is relevant for non-Gaussian models as well. Indeed, in the limit $g^{2} \rightarrow 0$, there will always be a value of $g^{2}$ sufficiently small that the model can be well approximated by a Gaussian model. Therefore the behavior of the 2-point functions in the transitory regime from dissipation to free propagation can be analyzed by studying Gaussian models (at least in the quasi-static limit).

\section{Appendix B: \\ Dissipative effects above $\Lambda_{L V}$. The Phenomenology}

We now have all the tools to construct models giving rise to dissipation in the vacuum above a critical energy scale $\Lambda_{L V}$. In this Appendix we work from a purely phenomenological point of view, and provide the class of dissipative models wherein the imaginary part of the self-energy is governed by a single term, in analogy with the dispersion relations of eq. (2).

From a phenomenological point of view, if one considers only stationary situations (i.e. static metrics and stationary states), one can simply choose the function $D(\omega, p)$ entering eq. (76) and eq. (80) as one wishes. There is indeed no restriction on $D(\omega, p)$ besides its constitutive properties, namely being odd in $\omega$ and giving rise to poles in $G_{r}$ all localized in the lower half $\omega$ plane. In this we have reached our first aim, namely identify how to generalize the free settings so as to incorporate some arbitrary dissipative effects.

We can thus consider the dispersive models which correspond to those defined by eq. (2). They are characterized by a single term giving rise to dissipation above $\Lambda_{L V}$. In the vacuum, they are fully specified by the imaginary part of the (retarded) self-energy

$$
-\operatorname{Im} \Sigma_{r}^{(n)}(\omega, p)=\frac{\omega}{\Lambda_{L V}} p^{2}\left(\frac{p}{\Lambda_{L V}}\right)^{2 n}=2 \omega \gamma_{n} .
$$

In these models, the decay rate (inverse life time) on the mass shell is

$$
\gamma_{n}=\frac{p}{2}\left(\frac{p}{\Lambda_{L V}}\right)^{2 n+1}
$$


To verify it, assuming that $\operatorname{Re} \Sigma_{r}=0$, the two poles of $G_{r}(\omega)$ in eq. (74) are located in

$$
\omega_{ \pm}(p)= \pm \sqrt{\omega_{p}^{2}-\gamma^{2}}-i \gamma
$$

From this, by inverse Fourier transform $G_{r}(\omega)$, one obtains that the decay rate is indeed $\gamma$ in the under-damped regime, for $\omega_{p}^{2}>\gamma^{2}$. In the overdamped regime, for $\gamma^{2}>\omega_{p}^{2}$, the decay rates of the two independent solutions of $G_{r}^{-1} \phi_{d}=0$ are $\Gamma_{ \pm}=\gamma \pm \sqrt{\gamma^{2}-\omega_{p}^{2}}$.

One thus have the following behavior as $p$ grows. For $p \ll \Lambda_{L V}, \omega_{ \pm} \simeq p$, and one has a free propagation which is slightly damped with a life time in the units of the frequency given by $\left(\Lambda_{L V} / \omega\right)^{n+1} \gg 1$. In the opposite regime of high momenta $p \gg \Lambda_{L V}$, deep in the overdamped regime, the two roots $\omega_{ \pm}$are real and the notion of propagation (in space-time) is absent. In anticipation to what will occur in inflation (or in black hole physics), we invite the reader to study the migration of the poles of $G_{r}$ when extrapolating backwards in time a mode, i.e. as $p$ increases. (Remember that the physical momentum of a mode in cosmology is $p_{\text {phys }}(t) \propto p / a(t)$ where $p$ is the norm of the conserved comoving wave vector (near a black hole one finds $p(r) \propto \omega / x$ where $x=r-r_{S}$ is the proper distance from the horizon measured in a freely falling frame, and $\omega$ the conserved Killing frequency.))

One could of course generalize the above class by considering in eq. (85) polynomials in $p$ dimensionalized by different UV scales. However, unless fine tuning, the phenomenology of the transition from the IR dissipation-free sector to the dissipative sector will be dominated a single term. One should also consider the possibility that $\operatorname{Im} \Sigma$ strictly vanishes below a certain frequency $\Omega_{1}$, as this would be the case when ever the spectrum of the $\Psi$ fields possesses this gap, see the remarks after eq. (76)).

Having the phenomenology of dissipative and unitary models under control (with dispersive and dissipative effects related by Kramers relation, eq. (77)) one could confront particle and astro-particle physics data and put lower bounds on $\Lambda_{L V}$ for each $n$, in analogy with what was done for (pure) dispersion in [1].

\section{References}

[1] T. Jacobson, S. Liberati and D. Mattingly, Annals Phys. 321 (2006) 150.

[2] V. F. Mukhanov, H. A. Feldman and R. H. Brandenberger, Phys. Rept. 215 (1992) 203.

[3] W. G. Unruh, Phys. Rev. D 51 (1995) 2827.

[4] T. Jacobson, Phys. Rev. D 48 (1993) 728.

[5] R. Brout, S. Massar, R. Parentani and P. Spindel, Phys. Rev. D 52 (1995) 4559, and R. Balbinot, A. Fabbri, S. Fagnocchi and R. Parentani, Riv. Nuovo Cim. 28 (2005) 1.

[6] J. Martin and R. H. Brandenberger, Phys. Rev. D 63 (2001) 123501.

[7] J. C. Niemeyer, Phys. Rev. D 63 (2001) 123502 . 
[8] J. C. Niemeyer and R. Parentani, Phys. Rev. D 64 (2001) 101301.

[9] M. V. Libanov and V. A. Rubakov, JCAP 0509 (2005) 005.

[10] M. V. Libanov and V. A. Rubakov, Phys. Rev. D 72 (2005) 123503.

[11] T. Jacobson, Phys. Rev. D 53 (1996) 7082.

[12] R. Parentani, "Constructing QFT's wherein Lorentz Invariance is broken by dissipative effects in the UV," arXiv:0709.3943 [hep-th].

[13] D. Arteaga, R. Parentani and E. Verdaguer, Phys. Rev. D 70 (2004) 044019, and Int. J. Theor. Phys. 46 (2007) 2227.

[14] R. Parentani, Int. J. Theor. Phys. 41 (2002) 2175 [arXiv:0704.2563], and Phys. Rev. D 63 (2001) 041503.

[15] P. C. Aichelburg and R. Beig, Annals of Physics, 98 (1976) 264.

[16] D. J. Raine, D. W. Sciama and P. G. Grove, Proc. R. Soc. A 435 (1991) 205.

[17] S. Massar, R. Parentani and R. Brout, Class. Quant. Grav. 10 (1993) 385.

[18] C. Gardiner and P. Zoller, "Quantum Noise," Springer (2000).

[19] W. G. Unruh and W. H. Zurek, Phys. Rev. D 40 (1989) 1071.

[20] D. Arteaga, arXiv:0711.0293 [quant-ph].

[21] N. D. Birrell and P. C. W. Davies, "Quantum fields in curved space," Cambridge University Press (1982).

[22] R. M. Wald, "Quantum field theory in curved space-time and black hole thermodynamics," Chicago, USA: Univ. Pr. (1994).

[23] B. Z. Foster and T. Jacobson, JHEP 0408 (2004) 024.

[24] A. Kempf, Phys. Rev. D 63 (2001) 083514.

[25] T. Jacobson, Phys. Rev. D 44 (1991) 1731.

[26] T. Jacobson and D. Mattingly, Phys. Rev. D 61 (2000) 024017.

[27] R. Parentani, C.R. Physique 4, 935 (2003), arXiv:astro-ph/0404022.

[28] W. G. Unruh, Phys. Rev. Lett. 46 (1981) 1351.

[29] U. H. Danielsson, Phys. Rev. D 66 (2002) 023511.

[30] J. C. Niemeyer, R. Parentani and D. Campo, Phys. Rev. D 66 (2002) 083510. 
[31] D. Campo, J. C. Niemeyer and R. Parentani, Phys. Rev. D 76 (2007) 023513

[32] D. S. Salopek and J. M. Stewart, Phys. Rev. D 47 (1993) 3235.

[33] R. Brout, S. Massar, R. Parentani, and Ph. Spindel, Phys. Rep. 260 (1995) 329.

[34] T. Jacobson, Prog. Theor. Phys. Suppl. 136 (1999) 1.

[35] C. Barrabès, V. Frolov, and R. Parentani, Phys. Rev. D62 (2000) 044020.

[36] K. Schalm, G. Shiu and J. P. van der Schaar, JHEP 0404 (2004) 076.

[37] J. R. Anglin, "Thermal equilibrium from the Hu-Paz-Zhang master equation," arXiv:hep-th/9210034.

[38] J. Adamek, D. Campo, J. Niemeyer, and R. Parentani, "Inflationary Spectra from Lorentz Violating Dissipative Models," to appear.

[39] J. Macher and R. Parentani, "Signatures of trans-Planckian dispersion in inflationary spectra," arXiv:0804.1920 [hep-th].

[40] T. Jacobson and D. Mattingly, Phys. Rev. D 63 (2001) 041502.

[41] M. Lemoine, M. Lubo, J. Martin and J. P. Uzan, Phys. Rev. D 65 (2002) 023510.

[42] S. Shankaranarayanan and M. Lubo, Phys. Rev. D 72 (2005) 123513. 